\title{
FLAVONOIDS IN THE SPECIES OF THE FAMILY ARACEAE: A REVIEW
}

\section{Sebuah tinjauan: Senyawa-senyawa flavonoid pada suku Araceae}

\author{
Tsukasa Iwashina \\ Tsukuba Botanical Garden, Department of Botany, National Museum of Nature and Science \\ Amakubo 4-1-1, Tsukuba, Ibaraki 305-0005 Japan \\ Email: iwashina@kahaku.go.jp
}

Diterima/Received: 25 Desember 2019; Disetujui/Accepted: 9 Maret 2020

\begin{abstract}
Abstrak
Flavonoid merupakan salah satu metabolit sekunder dan terdiri dari dua cincin fenil (cincin A dan B) yang dihubungkan oleh jembatan tiga karbon. Lebih dari 8000 jenis flavonoid dilaporkan dari tumbuhan berpembuluh dan Bryophyta. Makalah ini mengulas karakter flavonoid dari suku Araceae s.l. (termasuk Lemnaceae). Sebagian besar flavonoid utama dari suku Araceae adalah C-glikosilflavon dengan pengecualian untuk anak suku Gymnostachydoideae dan Orontioideae. Jenis flavonoid lainnya, yaitu anthocyanin, flavon, flavonol, flavan, dan proanthocyanidins dilaporkan terdapat pada semua anak suku Araceae. Flavanon dan C-glikosil flavanon diisolasi masing-masing dari Spirodela polyrhiza dan Anthurium binotii. Namun demikian, dihydroflavonol, chalcone, dihydrochalcone, aurone, dan isoflavonoid tidak dilaporkan terdapat pada jenis-jenis dari suku Araceae yang telah diteliti. Meskipun Araceae s.l. merupakan suku tumbuhan besar yang terdiri atas sekitar 3645 jenis dan 144 marga, baru 146 jenis dari 48 marga yang diketahui kandungan flavonoidnya.
\end{abstract}

Kata kunci: Araceae, C-glycosylflavone, distribusi, flavonoid, Lemnaceae

\begin{abstract}
Flavonoids is one of the secondary metabolites and consists of two phenyl rings ( $\mathrm{A}$ - and B-rings) connected by a three carbon bridge. Over 8000 kinds of flavonoids were reported from vascular plants and Bryophytes. This paper reviewed the flavonoid characters of the Araceae s.l. (including the Lemnaceae). Major flavonoids of the family were $C$-glycosylflavones with the exception for subfamilies Gymnostachydoideae and Orontioideae. Other flavonoid classes, i.e. anthocyanins, flavones, flavonols, flavan and proanthocyanidins, were reported in all subfamilies. Flavanone and C-glycosylflavanone were isolated from Spirodela polyrhiza and Anthurium binotii, respectively. However, dihydroflavonol, chalcone, dihydrochalcone, aurone, and isoflavonoids were not reported in the Araceae. Although Araceae s.l. is a large family consisting of ca. 3645 species and ca. 144 genera, flavonoids were surveyed only in 146 species of 48 genera, as far as known.
\end{abstract}

Keywords: Araceae, C-glycosylflavones, distribution, flavonoids, Lemnaceae

\section{INTRODUCTION}

Flavonoids is the general term for the compounds which have a fifteen carbon skeleton. At the simplest level, the skeleton consists of two phenyl rings ( $\mathrm{A}$ - and $\mathrm{B}$-rings) connected by a three carbon bridge (C-ring). In general, vascular plants and Bryophytes alone possess the biosynthetic ability of the flavonoids except for a few algae and fungi (List \& Freud 1968, Zeng et al. 2001, Liu et al. 2009). Flavonoids can be divided into several classes, e.g. anthocyanins, aurones, biflavones, chalcones, dihydrochalcones, dihydroflavonols, flavan and proanthocyanidins, flavanones, flavones, flavonols, isoflavonoids, and so on. Anthocyanins are based on the flavilium salt structure (Figure 1). The common anthocyanidins are pelargonidin, cyanidin, peonidin, delphinidin, petunidin, and 
malvidin. Flavones have substitutions on the A- and B-rings but lack oxygenation at the 3-position of the C-ring (Figure 2). Although flavones are generally present in vacuoles of cells as $O$ - and/or $C$ glycosides (C-glycosylflavones), some compounds particularly the simple and polymethoxylated flavones, occur in heart woods and as farinose exudates, bud wax, and so on. Flavonols are flavones that are attached to a hydroxyl group at 3position (Figure 3).

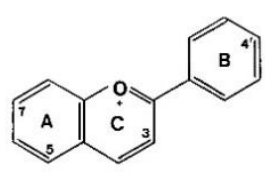

Figure 1. Anthocyanin<smiles>O=c1c(O)c(-c2ccccc2)oc2ccccc12</smiles>

Figure 3. Flavonol

Chalcones and dihydrochalcones lack a central heterocyclic ring (C-ring). Positions on these compounds are identified using a numbering system unique to these groups. Chalcones were apparently recognized as being structurally related to acetophenones whose ring carbons were identified by primed numbers. Hence, chalcones and dihydrochalcones A-ring carbons are also identified with primed numbers and the B-ring carbon is identified with unprimed numbers. Chalcones are double bonding between the $\alpha$ - and $\beta$-positions, but not in dihydrochalcones. So that the color of many chalcones turns yellow (Figure 4). Aurones are based on the 2-benzylidenecoumaranone or 2-benzylidene-3(2H)-benzofuranone system, and characterized by the presence of a five-membered heterocyclic ring. Aurone glycosides act as water-soluble yellow pigments on the flowers (Figure 5). Two structural features, i.e. the absence of the double bond between the 2- and 3-positions, and the presence of a chiral center at the 2-position, characterize flavanone. Dihydroflavonols, i.e. 3-hydroxyflavanones are requisite intermediates on the pathway to flavonols by one route and to anthocyanins via flavan 3,4-diols by another (Figure $6)$.<smiles>O=C(C=Nc1ccccc1)c1ccccc1</smiles><smiles>O=C(CCc1ccccc1)c1ccccc1</smiles>

Figure 4. Chalcone (upper) and Dihydrochalcone (lower)<smiles>O=C1C(=Cc2ccccc2)Oc2ccccc21</smiles>

Figure 5. Aurone<smiles>O=C1CC(c2ccccc2)Oc2ccccc2O1</smiles>

Figure 6. Flavanone (upper) and Dihydroflavonol (lower)
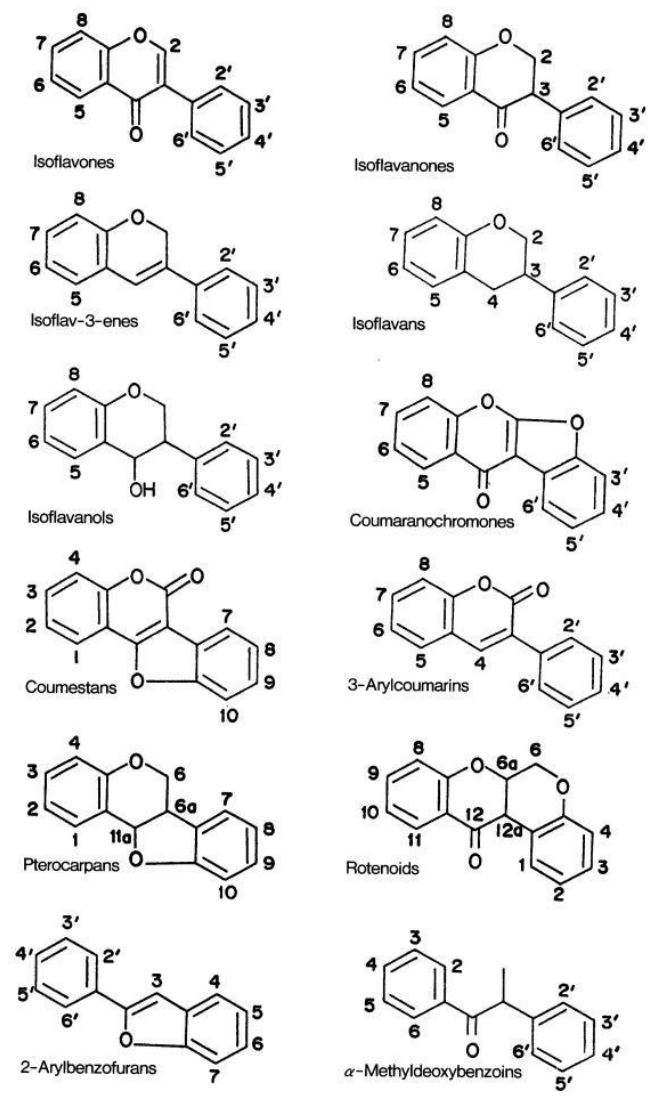

Figure 7. Basic chemical structures of isoflavonoids 
Isoflavonoids differ from other flavonoid classes in having as a basic structural feature that $B$ ring attaches to $\mathrm{C}-3$ but not $\mathrm{C}-2$, and subdivided into several classes, e.g. isoflavones, coumestans, coumaronochromones, pterocarpans, rotenoids, and so on (Figure 7). Flavan and proanthocyanidins which lack a 4-carbonyl group are noteworthy for their activities on human health. Moreover, numerous sorts of flavonoids occur in plants with additional hydroxyl, methoxyl, methyl and/or glycosyl substitution patterns. Additionally, aromatic and aliphatic acids, sulfate, prenyl and/or methylenedioxyl groups also attach to flavonoids and their glycosides. Thus, more than 8000 kinds of flavonoids have been reported as naturally occurring compounds. The isolation and identification, structures, distribution and biosynthesis of flavonoids in plants have been reviewed by many authors e.g. Harborne et al. (1975), Harborne \& Mabry (1982), Harborne (1988, 1994), Iwashina (2000), Andersen \& Markham (2006).

The flavonoids as medicinal resources were also reviewed by several researchers such as Cody et al. (1986, 1988), Rice-Evans \& Packer (1998). Anthocyanins in particular were recently noticed as antioxidants, antitumor, astringents as well as other medicinal properties (Ohba et al. 2000). However, secondary metabolites such as flavonoids were considered to be a waste products of plant metabolism in early days of the $20^{\text {th }}$ century. One of the most important functions of flavonoids may be to serve as an ultraviolet filter in land plants. It was shown by the survey of some plant species that the flavonoids act as UV shield.

The occurrence of anthocyanins as pollinator attractants is well-known as a function of flavonoids in plants. Additionally, it is known that flavones and flavonols, which can hardly be seen by human eyes, also act as pollinator attractants in addition to visible anthocyanins. Moreover, other functions, e.g. oviposition stimulants, feeding attractants, feeding deterrents, allelopathy and phytoalexins of naturally occurring flavonoids, were reported by many authors e.g. Iwashina (2003).

The Araceae is a large family of mostly herbaceous species, with great diverse in appearance. They are herbaceous with aerial stems or underground tubers or rhizomes, but there are a few woody species. The family included several climbers and epiphytes as well as a floating water plant, and consists of ca. 3645 species of ca. 144 genera (Boyce \& Croat 2011 onwards). APG III (2009) excluded the genus Acorus from Araceae, erected it in its own family, Acoraceae, and included Lemnaceae (genera Landoltia, Lemna, Spirodela, Wolffia, Wolffiella) into the Araceae. Araceae is divided into eight subfamilies, i.e. Aroideae, Gymnostachydoideae, Lasioideae, Lemnoideae, Monsteroideae, Orontioideae, Pothoideae, and Zamioculcadoideae. Of the araceous species, flavonoids were reported from 146 taxa of 48 genera. However, flavonoids were not reported from three genera of the Zamioculcadoideae growing in Africa. This review paper presents and describes the characters and distribution of flavonoids in the Araceae. The abbreviations used in Tables $1-8$ are as follows: ap = aerial part, $\mathrm{cr}=$ corm, ep = epidermis, $\mathrm{fl}=$ flower, $\mathrm{fr}=$ fruit, if = inflorescence, If = leaf, $\mathrm{pt}=$ petiole, $\mathrm{rz}=$ rhizome, $\mathrm{sp}$ $=$ spathe, $\mathrm{st}=$ stem, $\mathrm{sx}=$ spadix, $\mathrm{tb}=$ tuber, $\mathrm{wp}=$ whole plant.

\section{FLAVONOIDS IN THE SUBFAMILY GYMNOSTACHYDOIDEAE}

Gymnostachys anceps alone belongs to this subfamily and was surveyed for flavonoids (Table 1). A flavonol glycoside, kaempferol 3-sophoroside7-rhamnoside (Figure 8) was isolated from the leaves of this species (Williams et al. 1971). Other flavonoids were not reported. Williams et al. (1971) surveyed the $C$-glycosylflavones which were common flavonoids in the Araceae including Lemnaceae, however they were not present in this species.

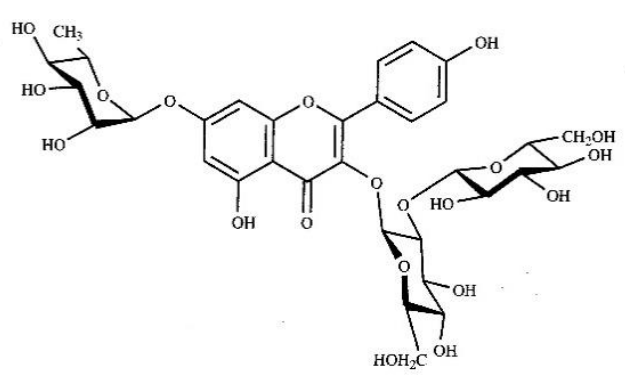

Figure 8. Kaempferol 3-sophoroside-7-rhamnoside

Table 1. Reports on the flavonoids from the species of subfamily Gymnostachydoideae

\section{Gymnostachys anceps R.Br.}

Flavonol: kaempferol 3-sophoroside-7-

rhamnoside (If) (Williams et al. 1971) 


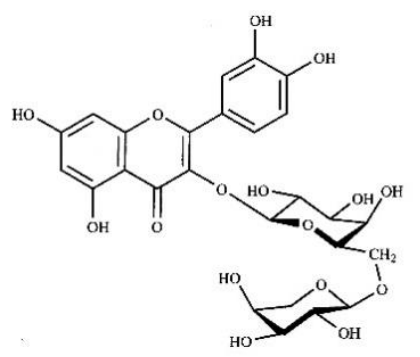

Figure 9. Quercetin 3-arabinosyl-(1 $\rightarrow 6)$ - galactoside

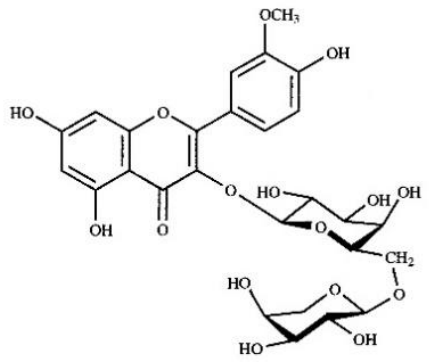

Figure 10. Isorhamnetin 3-arabinosyl-(1 $\rightarrow 6)$ galactoside

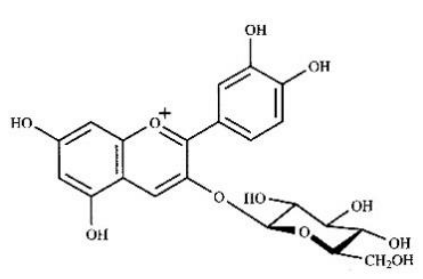

Figure 11. Cyanidin 3-glucoside

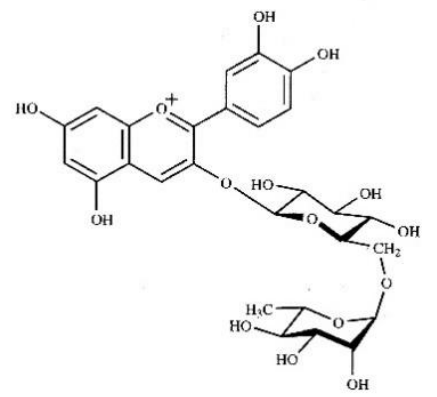

Figure 12. Cyanidin 3-rutinoside

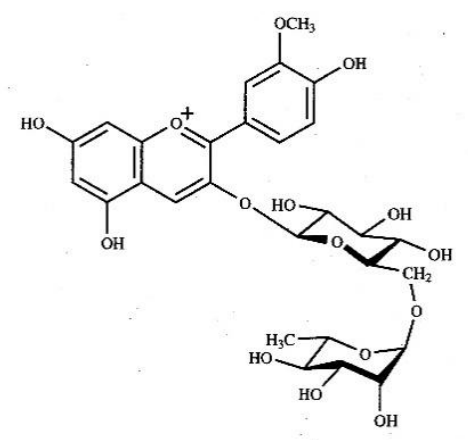

Figure 13. Peonidin 3-rutinoside

\section{FLAVONOIDS IN THE SUBFAMILY ORONTIOIDEAE}

Four species of subfamily Orontioideae, i.e. Lysichiton camtschatcensis, Orontium aquaticum, Symplocarpus foetidus, and S. renifolius, were surveyed for flavonoids (Table 2). Williams et al. (1981) and Whang \& Lee (1999) reported that major flavonoids of this subfamily were flavonols, namely, kaempferol 3-arabinosyl-(1-6)-galactoside, 3xylosylgalactoside and 3-sophoroside-7-glucoside, quercetin 3-arabinosyl-(1 $\rightarrow 6)$-galactoside (Figure 9), 3-galactoside, 3-sophoroside and 3sophoroside-7-glucoside, and isorhamnetin 3arabinosyl-(1 $\rightarrow 6)$-galactoside (Figure 10).

A quercetin glycoside which was acylated with caffeic acid, quercetin 3-sophoroside-7-(6"-Ecaffeoylglucoside), was isolated from the leaves of Symplocarpus renifolius (Whang \& Lee 1999). Three anthocyanins, namely cyanidin 3-glucoside (Figure 11), 3-rutinoside (Figure 12) and peonidin 3rutinoside (Figure 13), were detected in the flowers of Symplocarpus foetidus (Chang et al. 1970).
Table 2. Reports on the flavonoids from the species of subfamily Orontioideae

Lysichiton camtschatcensis (L.) Schott

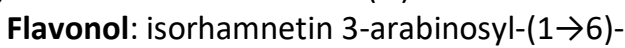
galactoside, kaempferol 3-arabinosyl- $(1 \rightarrow 6)$ galactoside, kaempferol 3-xylosylgalactoside, quercetin 3-arabinosyl-(1 $\rightarrow 6)$-galactoside (If) (Williams et al. 1981)

\section{Orontium aquaticum L.}

Flavonol: isorhamnetin 3-galactoside, isorhamnetin 3-rhamnosylgalactoside, kaempferol 3-galactosylglucoside, quercetin 3galactoside (If) (Williams et al. 1981)

Symplocarpus foetidus (L.) Salisb. ex W.P.C.Barton Anthocyanin: cyanidin 3-glucoside, cyanidin 3rutinoside, peonidin 3-rutinoside (fl) (Chang et al. 1970),

Flavonol: kaempferol 3-diglucoside, kaempferol galactosylglucoside, quercetin galactosylglucoside (If) (Williams et al. 1981)

\section{Symplocarpus renifolius Schott ex Tzvelev} Flavonol: isorhamnetin 3-sophoroside-7glucoside, kaempferol 3-sophoroside-7glucoside, quercetin 3-sophoroside, quercetin 3sophoroside-7-(6"'-E-caffeoylglucoside), quercetin 3-sophoroside-7-glucoside (If) (Whang \& Lee 1999) 
<smiles>O=c1cc(-c2ccc(O)cc2)oc2c(C(O)CC(O)C(O)C(O)CO)c(O)cc(O)c12</smiles>

Figure 14. Vitexin

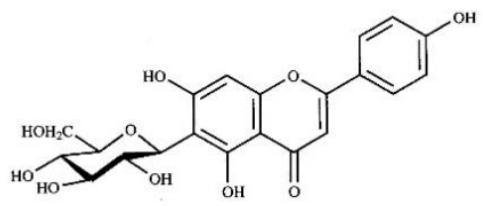

Figure 15. Isovitexin<smiles>O=c1cc(-c2ccc(O)c(O)c2)oc2c([C@H](O)[C@H](O)[C@H](O)[C@H](O)CO)c(O)cc(O)c12</smiles>

Figure 16. Orientin

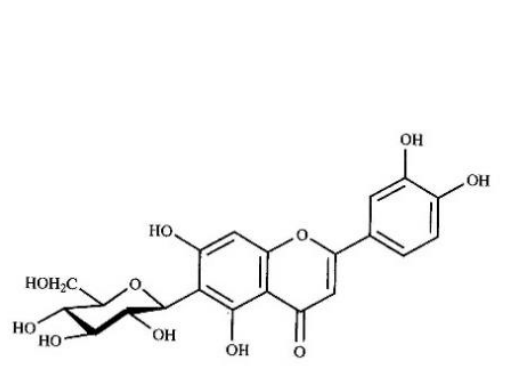

Figure 17. Isoorientin

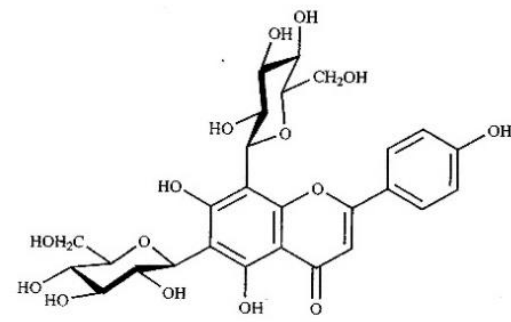

Figure 18. Vicenin-2

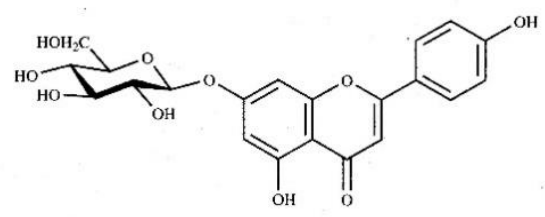

Figure 19. Apigenin 7-glucoside<smiles>CCC[C@H](O)[C@@H](O)[C@H](O)[C@H](O)Oc1cc(O)c2c(=O)cc(-c3ccc(O)c(O)c3)oc2c1</smiles>

Figure 20. Luteolin 7-glucoside

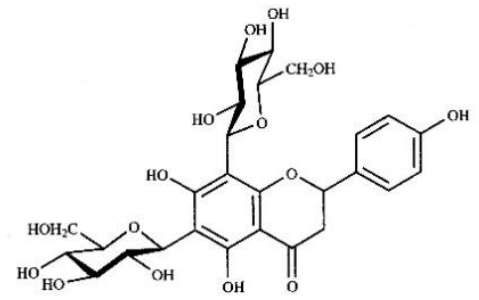

Figure 21. Naringenin 6,8-di-C-glucoside

\section{FLAVONOIDS IN THE SUBFAMILY LEMNOIDEAE}

Nineteen species of four genera of the Lemnoideae were surveyed for flavonoids (Table 3). The major flavonoid class of the subfamily is $C$ glycosylflavone. C-glycosylflavones such as vitexin (Figure 14), isovitexin (Figure 15), orientin (Figure 16), isoorientin (Figure 17), vicenin-2 (Figure 18) and their $O$-glycosides were found in all species surveyed, except for two Wolffia and three Wolffiella species. Major flavonoids in Wolffia microscopica and $W$. brasiliensis were flavonols, and kaempferol, quercetin and their 3-glycosides and 3,7-diglycoside were characterized (McClure \& Alston 1966). Major flavonoids in other Wolffia species, W. arrhiza, W. columbiana, W. globosa were flavones and $C$-glycosylflavones such as apigenin 7-glucoside (Figure 19), luteolin 7glucoside (Figure 20) (flavones), and vitexin, isovitexin, orientin, isoorientin (C-glycosylflavones) (McClure \& Alston 1966, Wang et al. 2014b).

Quercetin 3- and 3,7-diglycosides were found in three Wolffiella species (McClure \& Alston 1966). Various C-glycosylflavones, e.g. isovitexin, vitexin, orientin, isoorientin, vicenin-2, and their $O$ glycosides and acylated glycosides, were isolated from Lemna species, together with anthocyanin (cyanidin 3-glucoside) and flavones (McClure \& Alston 1966, Wallace \& Alston 1966, Veen 1975, Akhtar et al. 2010).

$C$-glycosylflavones frequently occured in Spirodela species with minor anthocyanins and flavonols. Another anthocyanin, petunidin 3,5diglucoside, was found in S. oligorrhiza (McClure \& Alston 1966). Rare $C$-glycosylflavanone, naringenin 6,8-di-C-glucoside (Figure 21), and Cglycosylflavone, 5,7-dihydroxy-3', 4' -methylenedioxyflavone 8 - $C$-glucoside, were isolated from S. polyrrhiza, together with two acylated $C$ glycosylflavones, apigenin 8-C-(2"'-feruloylglucoside) and luteolin 8-C-(2"'-feruloylglucoside) (Quiao et al. 2011). Two rare flavans, 3,5,4'trihydroxy-7,3'-dimethoxyflavan 5-glucoside and 3,5,4'-trihydroxy-4,7,3'-trimethoxyflavan 5-glucoside, were isolated from $S$. punctata (as Landoltia punctata), together with some common flavones and C-glycosylflavones (Wang et al. 2014a). 
Table 3. Reports on the flavonoids from the species of subfamily Lemnoideae

Lemna aequinoctialis Welw. (as Lemna trinervis

(Austin) Small) (McClure \& Alston 1966)

Flavone: luteolin, luteolin 7-glycoside (wp), C-Glycosylflavone: apigenin 6,8-di-C-glycoside, apigenin 6,8-di-C-glycoside (acylated), luteolin 6,8-di-C-glycoside (wp) (McClure \& Alston 1966)

\section{Lemna gibba L.}

Anthocyanin: cyanidin 3-glucoside (wp) (McClure \& Alston 1966),

Flavone: luteolin malonylglucosyl-malylglucoside (wp) (Akhtar et al. 2010),

C-Glycosylflavone: isoorientin, isoorientin 7glucoside, isovitexin, isovitexin $4^{\prime}$-glucoside, apigenin 6-C-(malonylglucoside)-glucosyl-malylglucoside, luteolin 6- $C$-(malonylglucoside)glucosyl-malylglucoside, orientin, vitexin (wp) (McClure \& Alston 1966, Veen 1975, Akhtar et al. 2010)

\section{Lemna japonica Landolt}

Flavone: chrysoeriol, luteolin 7-glucoside (wp), C-Glycosylflavone: isoorientin, isoorientin $2^{\prime \prime}-(E-$ caffeoyl-malate), isoorientin 2 "'-(E-p-coumaroylmalate), isoscoparin, isovitexin, isovitexin $2^{\prime \prime}-(E-$ caffeoyl-malate), lucenin-2, vicenin-2 (wp) (Bai et al. 2018)

\section{Lemna minor L.}

Flavone: apigenin 7-glycoside, chrysoeriol glucoside, isoscoparin, luteolin 7-glucoside (wp) (Wallace \& Alston 1966, Wallace et al. 1969, Vladimirova \& Georgiyants 2013),

C-Glycosylflavone: apigenin 6,8-di-C-glycoside, isoorientin, isoorientin 7-glucoside, isovitexin, isovitexin $4^{\prime}$-glucoside, luteolin 6,8-di-Cglycoside, orientin, vitexin (wp) (McClure \& Alston 1966, Wallace \& Alston 1966, Veen 1975)

Lemna minor (as Lemna minima Thuill. ex P.Beauv.) (McClure \& Alston 1966)

Flavone: apigenin 7-diglycoside, apigenin 7glycoside (wp),

C-Glycosylflavone: apigenin 6,8-di-C-glycoside, isoorientin, orientin (wp) (McClure \& Alston 1966)

Lemna obscura (Austin) Daubs

Anthocyanin: cyanidin 3-glucoside (wp), C-Glycosylflavone: apigenin 6,8-di-C-glycoside, apigenin 6,8-di- $C$-glycoside (acylated), isoorientin, luteolin 6,8-di-C-glycoside (acylated) (wp) (McClure \& Alston 1966)

\section{Lemna perpusilla Torr.}

Flavone: apigenin 7-glycoside (wp),

C-Glycosylflavone: apigenin 6,8-di-C-glycoside, apigenin 6,8-di-C-glycoside (acylated) (wp)

(McClure \& Alston 1966)

\section{Lemna trisulca $\mathrm{L}$.}

Anthocyanin: cyanidin 3-glucoside (wp), C-Glycosylflavone: apigenin 6,8-di-C-glycoside, apigenin 6,8-di-C-glycoside (acylated), isoorientin, luteolin 6,8 -di- $C$-glycoside, luteolin 6,8-di-C-glycoside (acylated), orientin, vitexin (wp) (McClure \& Alston 1966)

Lemna valdiviana Phil.

C-Glycosylflavone: apigenin 6,8-di-C-glycoside, apigenin 6,8-di-C-glycoside (acylated), luteolin 6,8-di-C-glycoside, luteolin 6,8-di- $C$-glycoside (acylated) (wp) (McClure \& Alston 1966)

Spirodela oligorrhiza (Kurz) Hegelm.

Anthocyanin: petunidin 3,5-diglucoside (wp), Flavone: luteolin, luteolin 7-diglycoside (wp), Flavonol: quercetin, quercetin 3,7-diglycoside (wp) (McClure \& Alston 1966),

C-Glycosylflavone: apigenin 6,8-di-C-glycoside, isoorientin, isoorientin 7-glucoside, isovitexin, isovitexin (acylated), isovitexin 7-glucoside, isovitexin 4'-glucoside, luteolin 6,8-di-Cglycoside, orientin (wp) (Jurd et al. 1957, McClure \& Alston 1966)

Spirodela polyrrhiza (L.) Schleid.

Anthocyanin: cyanidin 3-glucoside, cyanidin 3malonylglucoside (wp) (McClure \& Alston 1966, Reznik \& Menschick 1969, Krause \& Strack 1979), Flavanone: eriodictyol 7-glucoside, hesperetin 7glucoside (wp) (Quiao et al. 2011),

Flavone: apigenin, apigenin 7-glucoside, chrysoeriol, luteolin, luteolin diglucoside, luteolin 7-glucoside (wp) (McClure \& Alston 1966, Wallace \& Alston 1966, Reznik \& Menschick 1969, Wallace et al. 1969, Wallace 1975, Kim et al. 2010, Quiao et al. 2011),

Flavonol: quercetin diglucoside, quercetin diglucosylxyloside (wp),

C-Glycosylflavanone: naringenin 6,8-di-Cglucoside (wp) (Quiao et al. 2011),

C-Glycosylflavone: apigenin 8-C-(2"-feruloylglucoside), 5,7-Dihydroxy-3',4'- methylenedioxyflavone 8 -C-glucoside, isoorientin, isoorientin 7-glucoside, isovitexin, luteolin 8-C(2"'-feruloylglucoside), orientin, vitexin, vitexin 7glucoside (wp) (McClure \& Alston 1966, Wallace \& Alston 1966, Reznik \& Menschick 1969, Wallace et al. 1969, Saunders \& McClure 1976, 
Wallace 1975, Kim et al. 2010, Quiao et al. 2011)

Spirodela punctata (G.Mey.) C.H.Thomps. (as Landoltia punctata (G.Mey) Les \& D.J.Crawford) (Wang et al. 2014a)

Flavan and Proanthocyanidin: 3,5,4'-trihydroxy7,3'-dimethoxyflavan 5-glucoside, 3,5,4'-trihydroxy-4,7,3'-trimethoxyflavan 5-glucoside (wp),

Flavone: apigenin, apigenin 7-glucoside, luteolin, luteolin 7-glucoside (wp),

C-Glycosylflavone: apigenin 6-C-glucoside-8-Cgalactoside, isoorientin, isovitexin, orientin, vicenin-2, vitexin (wp) (Wang et al. 2014a)

Spirodela punctata (G.Mey.) C.H.Thomps. (as Spirodela biperforata W.Koch) (McClure \& Alston 1966)

Flavone: apigenin 7-glycoside, luteolin, luteolin 7-glycoside (wp),

C-Glycosylflavone: isoorientin, isoorientin 7glucoside, orientin, vitexin (wp) (McClure \& Alston 1966)

Spirodela punctata (as Spirodela intermedia W.Koch) (McClure \& Alston 1966, McClure 1968, Saunders \& McClure 1976)

Anthocyanin: cyanidin 3-glucoside (wp), Flavonol: kaempferol, kaempferol 3-glycoside, quercetin, quercetin 3-glycoside (wp) (McClure \& Alston 1966, McClure 1968),

C-Glycosylflavone: isovitexin 4'-glucoside, orientin, vitexin (wp) (McClure \& Alston 1966, McClure 1968, Saunders \& McClure 1976)

Wolffia arrhiza (L.) Horkel ex Wimm.

Flavone: luteolin, luteolin 7-diglycoside (wp), C-Glycosylflavone: apigenin 6,8-di-C-glycoside, Isoorientin, Isovitexin, orientin (wp) (McClure \& Alston 1966)

Wolffia brasiliensis Wedd. (as Wolffia papulifera C.H.Thomps.) (McClure \& Alston 1966)

Flavonol: kaempferol, kaempferol 3,7diglycoside, kaempferol 3-glycoside, quercetin, quercetin 3,7-diglycoside, quercetin 3-glycoside, quercetin 3,7-triglycoside (wp) (McClure \& Alston 1966)

Wolffia brasiliensis (Wolffia punctata Griseb.)

(McClure \& Alston 1966)

Flavonol: kaempferol, kaempferol 3,7-

diglycoside, kaempferol 3-glycoside, kaempferol

3,7-triglycoside, quercetin, quercetin 3-

diglycoside, quercetin 3-glycoside, quercetin 3,7triglycoside (wp) (McClure \& Alston 1966)
Wolffia columbiana H.Karst.

Flavone: luteolin, luteolin 7-diglycoside (wp), C-Glycosylflavone: apigenin 6,8-di-C-glycoside, isoorientin, isovitexin, orientin, vitexin (wp) (McClure \& Alston 1966)

Wolffia globosea (Roxb.) Hartog \& Plas

Flavone: apigenin 7-glucoside, luteolin 7-

glucoside (wp),

C-Glycosylflavone: isoorientin, isoorientin 6"glucoside, isovitexin, orientin, vicenin-2, vitexin (wp) (Wang et al. 2014b)

Wolffia microscopica (Griff.) Kurz

Flavonol: kaempferol, kaempferol 3,7-diglycoside, kaempferol 3-glycoside, quercetin, quercetin 3,7diglycoside, quercetin 3-glycoside, quercetin 3,7triglycoside (wp) (McClure \& Alston 1966)

Wolffiella gladiata (Hegelm.) Hegelm.

Flavonol: quercetin 3-glycoside, quercetin 3,7triglycoside (wp) (McClure \& Alston 1966)

Wolffiella gladiata (Wolffiella floridana (J.D.Sm.)

C.H.Thomps.) (McClure \& Alston 1966)

Flavonol: quercetin 3,7-triglycoside (wp)

(McClure \& Alston 1966)

Wolffiella lingulata (Hegelm.) Hegelm.

Flavonol: quercetin 3,7-diglycoside, quercetin 3glycoside, quercetin 3,7-triglycoside (wp) (McClure \& Alston 1966)

Wolffiella oblonga (Phil.) Hegelm.

Flavonol: quercetin 3,7-diglycoside, quercetin 3glycoside, quercetin 3,7-triglycoside (wp) (McClure \& Alston 1966)

\section{FLAVONOIDS IN THE SUBFAMILY POTHOIDEAE}

The subfamily Pothoideae consists of ca. 900 species of four genera. Twenty two species of two genera and Anthurium cultivars were surveyed for flavonoids (Table 4). Anthocyanins were found in the fruits, spathe and spadix of many Anthurium species and identified as cyanidin 3-glucoside, 3rutinoside, pelargonidin 3-rutinoside (Figure 22) and peonidin 3-rutinoside. Proanthocyanidins, procyanidins were also detected in some Anthurium species (Williams et al. 1981).

C-glycosylflavones flavones were isolated from three Anthurium species. They were relatively rare compounds, except for vitexin in $A$. versicolor, i.e. three embigenin (Figure 23) O-glycosides, 2"'- 
rhamnoside, 2"'-(4"'-3,4-dimethoxycinnamoylrhamnoside) and 2"'-(4"'-feruloylrhamnoside) from A. andraeanum (Clark et al. 2012), isoschaftoside (Figure 24) and schaftoside (Figure 25) from $A$. bellum (Williams et al. 1981), and cytisoside (Figure 26) 3"'-rhamnoside, and isocytisoside (Figure 27) 3"'-apiofuranoside, 3"'-rhamnoside and 6"'-xyloside from A. versicolor (Aquino et al. 2001). Crude extract of $A$. versicolor including their flavonoids was shown to have radical-scavenging activity (Aquino et al. 2001). Common flavonols, kaempferol and/or quercetin, were reported from four Anthurium species (Williams et al. 1981) and cultivars (Li et al. 2013).
Acacetin (Figure 28) was obtained from $A$. polyschistum (Williams et al. 1981), and rare methylated eucalyptin (Figure 29) and sideroxylin (Figure 30) glycosides were obtained from Anthurium cultivars (Li et al. 2013). A flavanone, hesperetin 7-rutinoside (Figure 31), was isolated from the epidermis of $A$. binotii (Brunswik 1921). From another Pothodeae species, Pothos chinensis, flavone glycoside, chrysoeriol (Figure 32) 7rhamnosylglucoside, and seven $C$-glycosyflavones, vitexin, vitexin 7-glucoside, isoschaftoside, schaftoside, isovitexin 7-glucoside, isoscoparin 7glucoside (Figure 33) and scoparin 7-glucoside (Figure 34), were isolated (Iwashina et al. 2010).

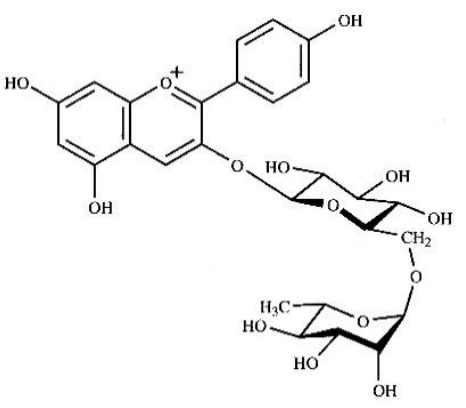

Figure 22. Pelargonidin 3-rutinoside

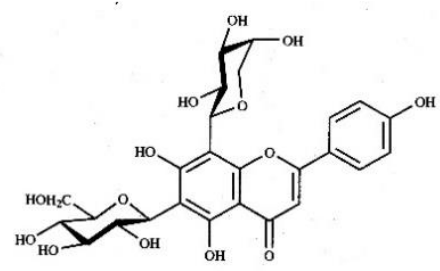

Figure 25. Schaftoside<smiles>COc1ccc(-c2cc(=O)c3c(O)cc(O)cc3o2)cc1</smiles>

Figure 28. Acacetin

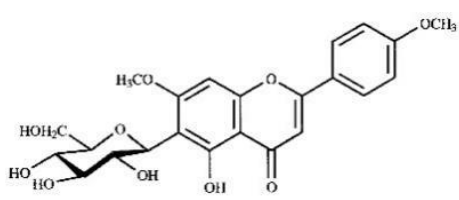

Figure 23. Embigenin<smiles>COc1ccc(-c2cc(=O)c3c(O)cc(O)c(CCOCCO)c3o2)cc1</smiles>

Figure 26. Cytisoside<smiles>COc1ccc(-c2cc(=O)c3c(O)c(C)c(OC)c(C)c3o2)cc1</smiles>

Figure 29. Eucalyptin

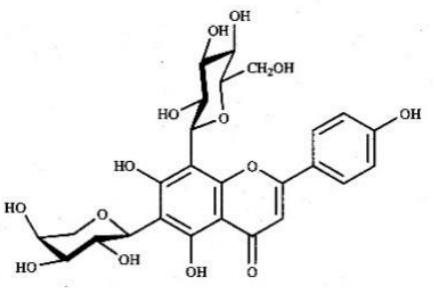

Figure 24. Isoschaftoside

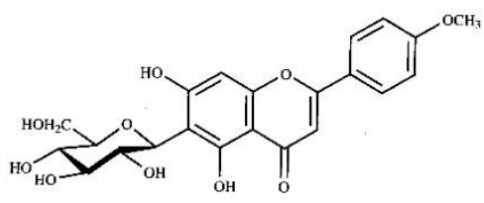

Figure 27. Isocytisoside

Figure 30. Sideroxylin

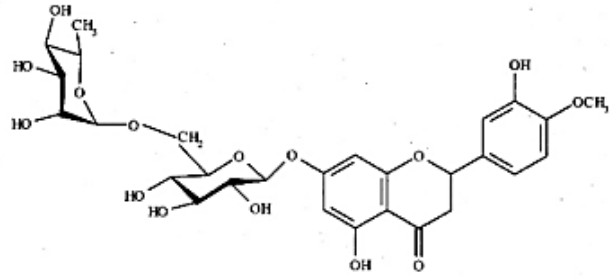

Figure 31. Hesperitin 7-rutinoside<smiles>COc1ccc(-c2cc(=O)c3c(O)cc(O)cc3o2)cc1O</smiles>

Figure 32. Chrysoeriol 


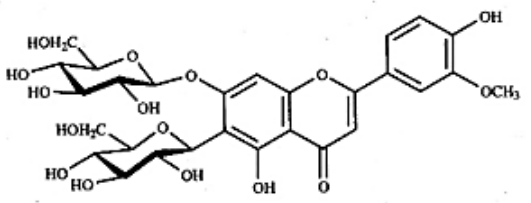

Figure 33. Isoscoparin 7-glucoside

Table 4. Reports on the flavonoids from the species of subfamily Pothoideae

Anthurium affine Schott

Anthocyanin: cyanidin 3-rutinoside, pelargonidin 3-rutinoside (fr) (Williams et al. 1981)

Anthurium andraeanum Linden ex André

Anthocyanin: cyanidin 3-rutinoside, pelargonidin 3-rutinoside (sp) (Iwata et al. 1979),

C-Glycosylflavone: embigenin 2"'-rhamnoside, embigenin 2"-(4"'-3,4- dimethoxycinnamoylrhamnoside), embigenin 2"'-(4"'-feruloyl-

rhamnoside) (If) (Clark et al. 2012)

\section{Anthurium bakeri Hook.f.}

Anthocyanin: cyanidin 3-rutinoside (fr) (Williams et al. 1981)

\section{Anthurium bellum Schott}

Anthocyanin: cyanidin 3-rutinoside, pelargonidin 3-rutinoside (sx),

Flavan and Proanthocyanidin: procyanidin (If), C-Glycosylflavone: isoschaftoside, schaftoside (If) (Williams et al. 1981)

\section{Anthurium binotii Linden}

Flavanone: hesperetin 7-rutinoside (ep)

(Brunswik 1921)

\section{Anthurium cultivars}

Anthocyanin: cyanidin 3-rutinoside, pelargonidin 3-rutinoside, peonidin 3-rutinoside (sp), Flavone: apigenin glucoside, chrysoeriol arabinosylglucoside, eucalyptin benzoylglucoside, luteolin glucoside, methylapigenin rhamnosylglucoside, methyleucalyptin benzoylglucoside, sideroxylin benzoylglucoside (sp),

Flavonol: kaempferol acetylmalonylglucoside, kaempferol 3-rhamnoside-7-(acetylarabinoside), kaempferol rhamnosylglucoside, quercetin rhamnoside (sp) (Li et al. 2013)

\section{Anthurium erskinei Mayo}

Anthocyanin: cyanidin 3-rutinoside (sp, sx) (Williams et al. 1981)

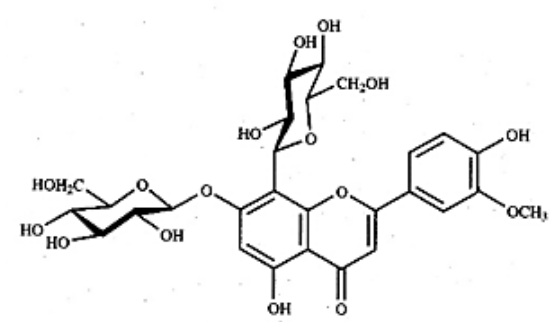

Figure 34. Scoparin 7-glucoside

Anthurium galeottii K.Koch

Anthocyanin: cyanidin 3-glucoside, cyanidin 3rutinoside, pelargonidin 3-rutinoside (sp) (Williams et al. 1981)

Anthurium gladiifolium Schott

Anthocyanin: cyanidin 3-rutinoside (sx), Flavan and Proanthocyanidin: procyanidin (If), Flavonol: kaempferol, quercetin (If) (Williams et al. 1981)

Anthurium gracile (Rudge) Lindl.

Anthocyanin: cyanidin 3-rutinoside, pelargonidin 3-rutinoside ( $\mathrm{fr}$ ),

Flavan and Proanthocyanidin: procyanidin (If)

(Williams et al. 1981)

Anthurium hookeri Kunth

Flavan and Proanthocyanidin: procyanidin (If) (Williams et al. 1981)

Anthurium inconspicuum N.E.Br.

Anthocyanin: cyanidin 3-rutinoside, pelargonidin 3-rutinoside (sp, sx) (Williams et al. 1981)

\section{Anthurium jilekii Schott}

Anthocyanin: cyanidin 3-rutinoside (sx) (Williams et al. 1981)

Anthurium lindmanianum Engl.

Flavan and Proanthocyanidin: procyanidin (If)

(Williams et al. 1981)

Anthurium longifolium (Hoffm.) G.Don

Anthocyanin: cyanidin 3-rutinoside (sp, sx)

(Williams et al. 1981)

Anthurium parasiticum (Vell.) Stellfeld (as Anthurium miquelianum K.Koch \& Augustin) (Williams et al. 1981)

Anthocyanin: cyanidin 3-rutinoside (pt, sx) (Williams et al. 1981)

Anthurium pentaphyllum (Aubl.) G.Don

Flavan and Proanthocyanidin: procyanidin (If), 
Flavonol: kaempferol, quercetin (If) (Williams et al. 1981)

Anthurium polyschistum R.E.Schult. \& Idrobo Flavone: acacetin (If) (Williams et al. 1981)

\section{Anthurium radicans K.Koch \& Haage}

Anthocyanin: cyanidin 3-rutinoside (pt), Flavan and Proanthocyanidin: procyanidin (If), Flavonol: kaempferol, quercetin (If) (Williams et al. 1981)

\section{Anthurium regale Linden}

Anthocyanin: cyanidin 3-rutinoside (sx), Flavan and Proanthocyanidin: procyanidin (If), Flavonol: quercetin (If) (Williams et al. 1981)

\section{Anthurium schlechtendalii Kunth}

Anthocyanin: cyanidin 3-rutinoside (fr) (Williams et al. 1981)

\section{Anthurium versicolor Sodiro}

C-Glycosylflavone: cytisoside 3"'-rhamnoside, isocytisoside 3"'-apiofuranoside, isocytisoside 3"rhamnoside, isocytisoside 6"-xyloside, vitexin (If) (Aquino et al. 2001)

Pothos chinensis (Raf.) Merr.

Flavone: chrysoeriol 7-rhamnosylglucoside (ap), C-Glycosylflavone: isoschaftoside, isoscoparin 7glucoside, isovitexin 7-glucoside, schaftoside, scoparin 7-glucoside, vitexin, vitexin 7-glucoside (ap) (Iwashina et al. 2010)

\section{FLAVONOIDS IN THE SUBFAMILY MONSTEROIDEAE}

The Monsteroideae consists of ca. 360 species and 12 genera. Only one species, Scindapsus pictus, was surveyed for its flavonoids. Flavone (chrysoeriol) and flavonol (quercetin) were detected (Table 5) (Williams et al. 1981).

Table 5. Reports on the flavonoids from the species of subfamily Monsteroideae

Scindapsus pictus Hassk.

Flavone: chrysoeriol (If),

Flavonol: quercetin (If) (Williams et al. 1981)

\section{FLAVONOIDS IN THE SUBFAMILY LASIOIDEAE}

About 60 species of 10 genera belong to the Lasioideae. Two species, Dracontium asperum and Lasia spinosa, were surveyed for flavonoids (Table 6). Common anthocyanins, cyanidin and pelargonidin 3-rutinosides, were found in Dracontium asperum (Williams et al. 1981). CGlycosylflavones were detected in $D$. asperum and L. spinosa, and characterized as isovitexin xyloside and vitexin glucoside (D. asperum) (Williams et al. 1981), and vitexin and its 2"-glucoside (L. spinosa) (Hong Van et al. 2006).

Table 6. Reports on the flavonoids from the species of subfamily Lasioideae

\section{Dracontium asperum K.Koch}

Anthocyanin: cyanidin 3-rutinoside, pelargonidin 3-rutinoside (pt),

C-Glycosylflavone: isovitexin xyloside, vitexin glucoside (If) (Williams et al. 1981)

Dracontium asperum (as Dracontium foecundum Hook.f.) (Williams et al. 1981)

Anthocyanin: cyanidin 3-rutinoside, pelargonidin 3-rutinoside (sp, sx) (Williams et al. 1981)

Lasia spinosa (L.) Thwaites

Flavonol: quercetin 3-rutinoside (wp),

C-Glycosylflavone: vitexin, vitexin 2"'-glucoside (wp) (Hong Van et al. 2006)

\section{FLAVONOIDS IN THE SUBFAMILY AROIDEAE}

The Aroideae is the largest subfamily of Araceae and consists of 70 genera. Flavonoids from 94 species of 35 genera were surveyed (Table 7). Of seven surveyed Alocasia species, anthocyanin, cyanidin 3-rutinoside was found in five species. Procyanidins and common flavonols, kaempferol and/or quercetin, were accompanied by anthocyanin in almost species (Williams et al. 1981). An acylated anthocyanin was isolated from the tubers of $A$. cucullata and identified as cyanidin 3(6"- $E$-p-coumaroylglucoside)-5-(6"'-malonyl-glucoside) (Figure 35) (Lei et al. 2014).

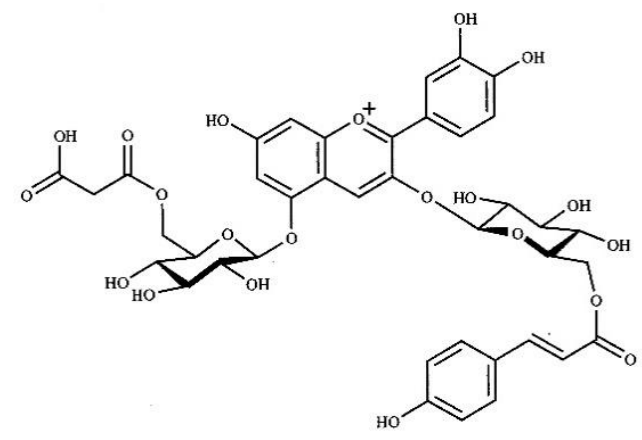

Figure 35. Cyanidin 3-(6"-E-p-coumaroylglucoside)-5(6"-malonylglucoside) 
Five Amorphophallus species were surveyed for their flavonoids. Of these species, A. titanum is the most well-known as the plant having the largest inflorescence among the plant kingdom, and the flavonoids of its spathe, and spadix and leaves were analyzed. The major flavonoids were anthocyanins and $C$-glycosylflavones, and identified as cyanidin 3glucoside and 3-rutinoside, pelargonidin 3coumaroylglucoside and 3-rhamnosylglucoside and peonidin 3-coumaroylglucoside, 3-glucoside and 3rutinoside as anthocyanins, and isoorientin, orientin, schaftoside, isoschaftoside, vicenin-2, lucenin-2, vitexin and its 2"-glucoside, and isovitexin and its 2"'-glucoside and X"'-rhamnoside (Gallori et al. 2004, Iwashina et al. 2015, 2020). Two flavones and five flavonols were accompanied by $C$ glycosylflavones and identified as chrysoeriol 7glucoside and luteolin 7-glucoside, and kaempferol 3-robinobioside (Figure 36), 3-rhamnosylarabinoside and 3-rutinoside, and quercetin 3robinobioside and 3-rutinoside (Iwashina et al. 2015, 2020). Although similar anthocyanins, Cglycosylflavones and flavonols were obtained from the other two Amorphophallus species, i.e. A. paeoniifolius and A. konjac (Iwashina et al. 2015), a rare flavonol, 3,5-diacetyltambulin (7,8,4'trimethoxy-3,5-diacetylflavone) was found in the former species (Khan et al. 2008). This flavonoid showed significant antibacterial activities against four Gram-positive bacteria (e.g. Bacillis subtilis and Staphylococcus aureus) and six Gram-negative bacteria (e.g. Escheichia coli, Shigella sonnei, Pseudomonas aerginosa, and Salmonella typhi) (Khan et al. 2008).

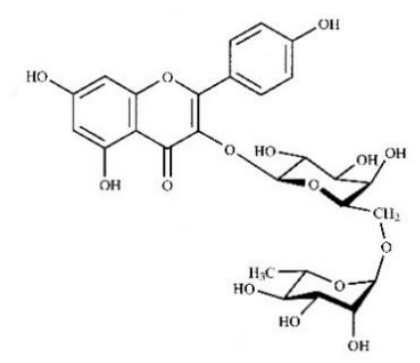

Figure 36. Kaempferol 3-robinobioside

Three $C$-glycosylflavones, apigenin 6,8-di-Cgalactoside (Figure 37), isocorymboside (Figure 38) and neocorymboside (Figure 39), were isolated from the rhizomes and tubers of Arisaema erubescens, together with isoschaftoside, schaftoside and vicenin-2 (Du et al. 2005, 2011). Of their compounds, isoschaftoside and schaftoside showed the strong nematicidal activity against the root-knot nematode (Meloidogyne incognita) (Du et al. 2011).

Seven Arum taxa were surveyed for flavonoids. Although common $C$-glycosyflavones such as isovitexin, vitexin, isoorientin, orientin and rarely their $\mathrm{O}$-glucosides were major flavonoids in Arum species, flavones e.g. apigenin, luteolin and chrysoeriol, flavonols e.g. quercetin and its 3glycosides, and anthocyanins, cyanidin 3 -glucoside and 3-rutinoside were scattered present (Phouphas 1956, Williams et al. 1981, Koleva 1982, 1984, Afifi et al. 2016). Polymethoxylated flavonol, quercetin $7,3^{\prime}, 4^{\prime}$-trimethyl ether (Figure 40), was isolated from the aerial parts of Arum palaestinum (Farid et al. 2015). Of the flavonoids from A. palaestinum, each two of flavones and $C$-glycosylflavones, i.e. luteolin and chrysoeriol, and isoorientin and isovitexin, showed a significant high antiproliferative activity (Farid et al. 2015).

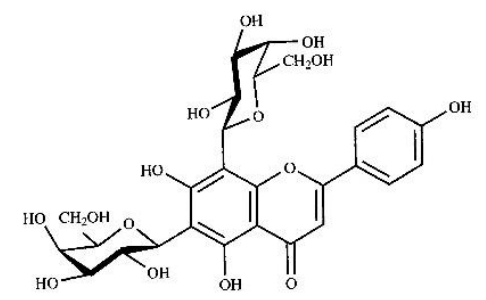

Figure 37. Apigenin 6,8-di-C-galactoside

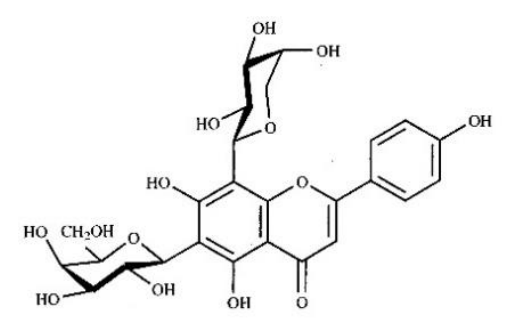

Figure 38. Isocorymboside

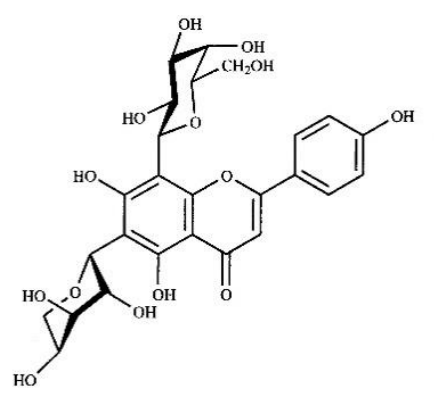

Figure 39. Neocorymboside 
<smiles>COc1ccc(-c2oc3cc(O)cc(O)c3c(=O)c2C)cc1O</smiles>

Figure 40. Quercetin 7,3',4'-trimethyl ether

Six rare C-glycosylflavones were isolated from the leaves of Asterostigma riedelianum. They were characterized as apigenin $7,4^{\prime}$-dimethyl ether 6-C-arabinoside (Figure 41), 6,8-di- $C$-arabinoside (Figure 42), 6- $C$-arabinoside-2"-glucoside (Figure 43) and 6-C-arabinoside-2"-caffeoylglucoside, and isomolludistin 2"-glucoside (Figure 44) and 2"caffeoylglucoside (Markham \& Williams 1980).

Colocasia esculenta is widely cultivated in the world especially in tropical zone for its tubers (known as "taro"). The species was fully analyzed for flavonoids. Major flavonoids are $C$ glycosylflavones together with minor flavones, and many compounds were isolated, e.g. isoschaftoside, schaftoside, vicenin-2, isoorientin, orientin and its 7-glucoside, isovitexin and its 4'glucoside, and apigenin, chrysoeriol, diosmetin, luteolin $O$-glycosides and so on (Iwashina et al. 1999, Leong et al. 2010, Ferreres et al. 2012, Li et al. 2014). Of their C-glycosylflavones, orientin and isoorientin significantly inhibited rat lens aldose reductase (Li et al. 2014). Anthocyanins, cyanidin 3glucoside and 3-rhamnoside, and pelargonidin 3glucoside, flavones, chrysoeriol and luteolin 7glycosides, procyanidins and flavonol, quercetin, were accompanied by $\mathrm{C}$-glycosylflavones, were also found in this species (Chan Jr. \& Kao-Jao 1977, Williams et al. 1981, Iwashina et al. 1999).<smiles>COc1ccc(-c2cc(=O)c3c(O)c(C4CC(O)C[C@@H](C)O4)c(O)cc3o2)cc1</smiles>

Figure 41. Apigenin 7,4'-dimethyl ether 6 - $C$-arabinoside

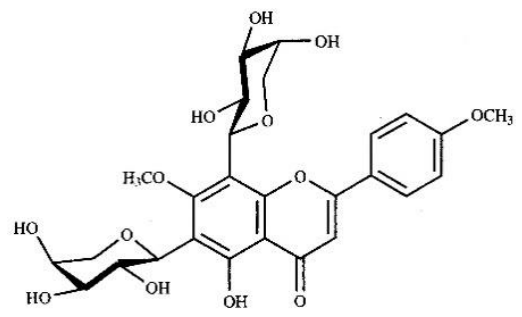

Figure 42. Apigenin 7,4'-dimethyl ether $6,8-$ di- $C$ arabinoside

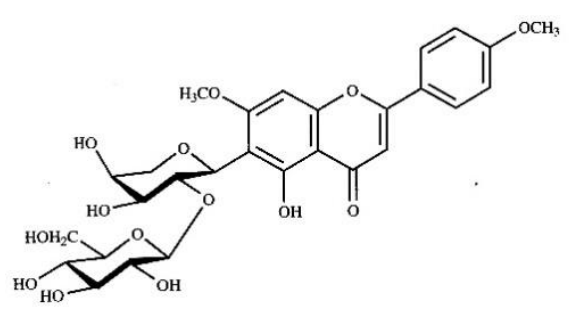

Figure 43. Apigenin 7,4'-dimethyl ether $6-C$ arabinoside-2"-glucoside

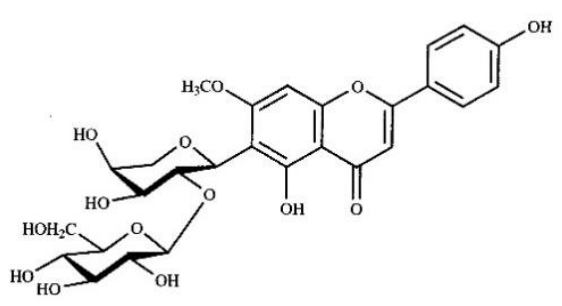

Figure 44. Isomolludistin 2"-glucoside

Nine Cryptocoryne species were surveyed for flavonoids. Vitexin 2"'-glucoside (Figure 45) and sometimes 2"-glucoside-6"-E-sinapate were isolated from all species, except for $C$. wendtii (Franke et al. 2006). A rare sulfated Cglycosylflavone was isolated from Culcasia saxatilis and identified as vitexin 7-sulfate (Figure 46) (Williams et al. 1981). Two sulfated Cglycosylflavones were also isolated from Philodendron ornatum and identified as vitexin 7sulfate and isovitexin 7-sulfate (Williams et al. 1981). Although 26 Philodendron species were surveyed for flavonoids and anthocyanins such as cyanidin 3-glycosides and rarely delphinidin, flavonols such as kaempferol, quercetin and isorhamnetin and procyanidins were found. They were roughly analyzed, except for $P$. undulatum containing four $C$-glycosylflavones (isoorientin, orientin, isoschaftoside and schaftosife) and $P$. saxicola containing four flavonols (isorhamnetin 3glucoside, isorhamnetin 3-rutinoside, quercetin 3glucoside and quercetin 3-rutinoside), and apigenin and luteolin C-glycosides (Williams et al. 1981). 


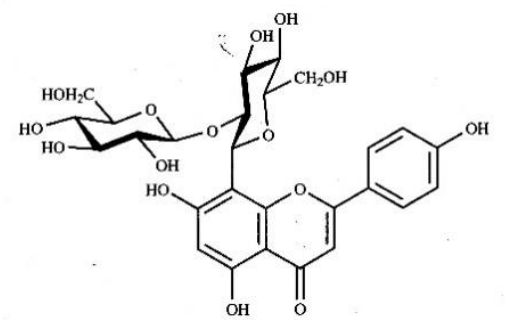

Figure 45. Vitexin 2"-glucoside<smiles>O=c1cc(-c2ccc(O)cc2)oc2c(C(O)C(O)CCO)c(OS(=O)(=O)[O-])cc(O)c12</smiles>

Figure 46. Vitexin 7-sulfate

Aquatic weed, Pistia stratiotes, contained flavones, chrysoeriol 4'-glucoside (Figure 47), luteolin and its 7-glycoside, and C-glycosylflavones, vitexin, orientin, and apigenin, luteolin 6,8-di-Cglycosides, and anthocyanin, cyanidin 3-glucoside (Zennie \& McClure 1977, Liu et al. 2008, Tripathi et al. 2016). Six C-glycosylflavones, vitexin, vicenin-2, apigenin 6 - $C$-glucoside-8-C-apiofuranoside, and isovitexin and its $6^{\prime \prime}$-glucoside and $4^{\prime}$-rhamnoside, were isolated from the leaves of Xanthosoma sagittifolium, together with anthocyanin, cyanidin 3-rutinoside (Williams et al. 1981, Picerno et al. 2003). The fraction containing their $C$ glycosylflavones showed a significant antioxidant/ free-radical scavenging activity (Picerno et al. 2003). Six $C$-glycosylflavones were isolated from Zantedeschia aethiopica, together with other flavonoids, apigenin, luteolin, kaempferol and quercetin, and identified as isoorientin, isovitexin, orientin, swertiajaponin (Figure 48), swertisin (Figure 49) and vitexin (Martens et al. 2003, Luzzatto et al. 2007, Nakayama et al. 2015). Of their $C$-glycosylflavones, isoorientin and swertiajaponin responded to low temperature-induced yellow pigmentation of the bracts of this species (Nakayama et al. 2015). Moreover, swertisin and isovitexin showed the antimicrobial activity against Pectobacterium carotovorum (Luzzatto et al. 2007).

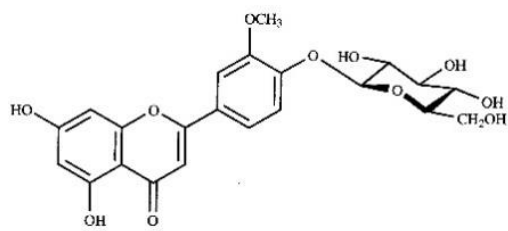

Figure 47. Chrysoeriol 4'-glucoside

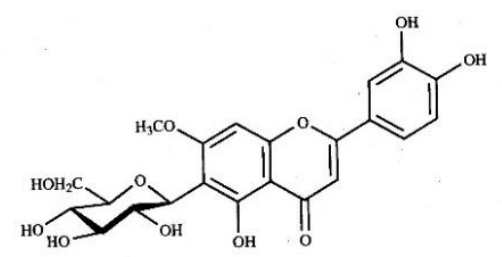

Figure 48. Swertiajaponin

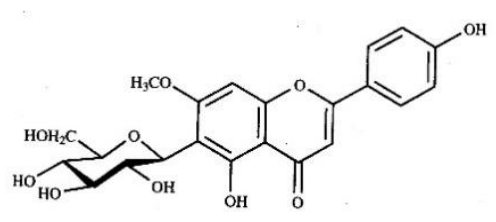

Figure 49. Swertisin

Williams et al. (1981) analyzed the flavonoids of many Aroideae species, e.g. Aglaonema modestum, Alocasia spp., Anchomanes spp. Anubias varteri var. glabra, Apoballis acuminatissima, Arophyton crassifolium, Caladium bicolor, Calla palustris, Carlephyton spp., Cercestis spp., Dracunculus spp., Eminium spp., Helicodiceros muscivorus, Homalomena spp., Peltandra virginica, Pinellia tripartita, Stylochaeton spp., Synandrospadix vermitoxicus, Syngonium spp., Typhonium flagelliforme, and Typhonodorum lindleyanum. Although anthocyanins, flavones, flavonols and proanthocyanidins were reported, they were insufficiently identified.

Table 7. Reports on the flavonoids from the species of subfamily Aroideae

Aglaonema modestum Schott ex Engl.

Flavan and Proanthocyanidin: procyanidin (If) (Williams et al. 1981)

Alocasia cucullata (Lour.) G.Don

Anthocyanin: cyanidin 3-(6"'-E-p-coumaroylglucoside)-5-(6"'-malonylglucoside) (tb) (Lei et al. 2014)

\section{Alocasia cuprea K.Koch}

Anthocyanin: cyanidin 3-rutinoside (If),

Flavonol: kaempferol, quercetin (If) (Williams et al. 1981) 
Alocasia lauterbachiana (Engl.) A.Hay (as Xenophya lauterbachiana (Engl.) Nicolson) (Williams et al. 1981)

Anthocyanin: cyanidin 3-rutinoside (If),

Flavonol: quercetin (If) (Williams et al. 1981)

Alocasia longiloba Miq. (as Alocasia thibantiana Mast.) (Williams et al. 1981)

Anthocyanin: cyanidin 3-rutinoside (If), Flavonol: kaempferol, quercetin (If) (Williams et al. 1981)

Alocasia macrorrhizos (L.) G.Don (as Alocasia macrorrhizos var. rubra (Hassk.) Furtado) (Williams et al. 1981)

Anthocyanin: cyanidin 3-rutinoside (pt), Flavan and Proanthocyanidin: procyanidin (If), Flavonol: quercetin (If) (Williams et al. 1981)

Alocasia macrorrhizos (as Alocasia macrorrhizos var. variegata (K.Koch \& C.D.Bouché) Furtado) (Williams et al. 1981)

Anthocyanin: cyanidin 3-rutinoside (pt), Flavan and Proanthocyanidin: procyanidin (If), Flavonol: kaempferol, quercetin (If) (Williams et al. 1981)

Alocasia macrorrhizos (as Colocasia indica (Lour.) Kunth) (Williams et al. 1981)

Flavan and Proanthocyanidin: procyanidin (If) (Williams et al. 1981)

Alocasia odora (Lindl.) K.Koch

Flavan and Proanthocyanidin: procyanidin (If) (Williams et al. 1981)

Alocasia portei Schott

Anthocyanin: cyanidin 3-rutinoside (pt) (Williams et al. 1981)

Amorphophallus abyssinicus (A.Rich.) N.E.Br. Anthocyanin: cyanidin 3-rutinoside (sp) (Williams et al. 1981)

\section{Amorphophallus konjac K.Koch}

Anthocyanin: cyanidin 3-glucoside, cyanidin 3rutinoside, peonidin 3-glucoside, peonidin 3rutinoside, pelargonidin 3-rhamnosylglucoside (if),

Flavonol: quercetin 3-glucoside (if), C-Glycosylflavone: isoorientin, isovitexin, orientin, vitexin 2"'-xyloside (if) (Iwashina et al. 2015)
Amorphophallus paeoniifolius (Dennst.) Nicolson

(Amorphophallus campanulatus Decne)

Anthocyanin: cyanidin 3-glucoside, cyanidin 3-

diglucoside, pelargonidin 3-glucoside (if)

(Iwashina et al. 2015),

Flavonol: 3,5-diacetytambulin, kaempferol 3-

glucoside, quercetin, quercetin 3-glucoside (cr, if, tb) (Khan et al. 2008, Sharstry et al. 2010,

Iwashina et al. 2015),

C-Glycosylflavone: isovitexin, orientin, schaftoside, vicenin-2, vitexin (if) (Iwashina et al. 2015)

Amorphophallus stuhlmannii (Engl.) Engl. \& Gehrm. Anthocyanin: cyanidin 3-rutinoside (sp) (Williams et al. 1981)

Amorphophallus titanum (Becc.) Becc.

Anthocyanin: cyanidin 3-glucoside, cyanidin 3rutinoside, pelargonidin 3-coumaroylglucoside, pelargonidin 3-rhamnosylglucoside, peonidin 3coumaroylglucoside, peonidin 3-glucoside, peonidin 3-rutinoside (sp, sx) (Gallori et al. 2004, Iwashina et al. 2015),

Flavone: chrysoeriol 7-glucoside (sp, sx) (Iwashina et al. 2015), luteolin 7-glucoside (If) (Iwashina et al. 2020),

Flavonol: kaempferol 3-robinobioside, kaemopferol 3-rhamnosylarabinoside, kaempferol 3-rutinoside, quercetin 3robinobioside, quercetin 3-rutinoside (If) (Iwashina et al. 2020),

C-Glycosylflavone: isoscoparin $X^{\prime \prime}$-glucoside, isovitexin 2 "'-glucoside, isovitexin $X^{\prime \prime}$ rhamnoside, vitexin 2"-glucoside (sp, sx) (Iwashina et al. 2015), isoorientin, isoschaftoside, isovitexin, lucenin-2, orientin, schaftoside, vicenin-2, vitexin (sp, sx, If) (Iwashina et al. 2015, 2020)

Anchomanes abbreviatus Engl.

Anthocyanin: cyanidin 3-gentiobioside, pelargonidin 3-gentiobioside, pelargonidin 3glucoside (fr) (Williams et al. 1981)

Anchomanes difformis (Blume) Engl. Flavan and Proanthocyanidin: procyanidin (If) (Williams et al. 1981)

Anubias barteri Schott var. glabra N.E.Br. (Anubias lanceolata N.E.Br.) (Williams et al. 1981)

Flavan and Proanthocyanidin: procyanidin (If) (Williams et al. 1981) 
Apoballis acuminatissima (Schott) S.Y.Wong \& P.C.Boyce (as Schismatoglottis concinna Schott var. immaculata N.E.Br.) (Williams et al. 1981)

Anthocyanin: delphinidin 3-rutinoside (If, pt), Flavan and Proanthocyanidin: procyanidin (If), Flavonol: isorhamnetin, quercetin (If) (Williams et al. 1981)

\section{Arisaema erubescens (Wall.) Schott}

C-Glycosylflavone: apigenin 6,8-di-C-galactoside, isocorymboside, isoschaftoside, neocorymboside, schaftoside, vicenin-2 (rz, tb) (Du et al. 2005, 2011)

Arisaema serratum (Thunb.) Schott Anthocyanin: cyanidin 3,5-diglycoside (sp) (Ueno et al. 1969)

Arisarum vulgare O.Targ.Tozz. C-Glycosylflavone: orientin, vitexin (If) (Pagani 1982)

Arophyton crassifolium (Buchet) Bogner Flavan and Proanthocyanidin: procyanidin (If) (Williams et al. 1981)

Arum dioscoridis Sm.

Flavone: apigenin, luteolin (fl, If),

Flavonol: quercetin, quercetin 3-glucoside (fl, If), C-Glycosylflavone: isoorientin, vitexin (fl, If) (Afifi et al. 2016)

Arum italicum Mill. subsp. italicum

Flavone: chrysoeriol, luteolin (If) (Williams et al. 1981),

C-Glycosylflavone: isovitexin 7-glucoside (rz)

(Phouphas 1956)

Arum italicum subsp. neglectum (F.Towns.) Prime Flavone: chrysoeriol (If) (Williams et al. 1981)

\section{Arum maculatum L.}

Anthocyanin: cyanidin 3-glucoside, cyanidin 3rutinoside (sp, sx),

Flavone: chrysoeriol 7-glucoside, luteolin 7glucoside (If),

C-Glycosylflavone: apigenin di-C-glycoside, isoorientin, isovitexin, luteolin di-C-glycoside, orientin (If) (Williams et al. 1981)

Arum orientale M.Bieb.

Flavone: apigenin (If),

Flavonol: quercetin, quercetin 3-rhamnoside, quercetin 3-rutinoside (If) (Koleva 1984), C-Glycosylflavone: isoorientin, isovitexin, isovitexin 7-glucoside, orientin, vitexin (If) (Koleva 1982, 1984)

\section{Arum palaestinum Boiss.}

Flavone: apigenin, chrysoeriol, luteolin (ap, fl, If), Flavonol: quercetin, quercetin 7,3',4'-trimethyl ether (ap, fl, If) (Farid et al. 2015, Afifi et al. 2016),

C-Glycosylflavone: isoorientin, isovitexin, vitexin (ap, fl, If) (Afifi et al. 1999, 2016, Farid et al. 2015)

\section{Asterostigma riedelianum (Schott) Kuntze}

Anthocyanin: cyanidin 3-glucoside, cyanidin 3rutinoside (st) (Williams et al. 1981),

C-Glycosylflavone: apigenin 7,4'-dimetyl ether 6$c$-arabinoside, apigenin 7,4' -dimethyl ether 6,8di- $C$-arabinoside, apigenin 7,4' -dimethyl ether 6 $C$-arabinoside-2"'-glucoside, apigenin 7,4' dimethyl ether 6-C-arabinoside-2"-(caffeoylglucoside), isomolludistin 2"'-(caffeoylglucoside), isomolludistin 2"'-glucoside (If) (Markham \& Williams 1980, Williams et al. 1981)

Biarum tenuifolium (L.) Schott

Flavone: chrysoeriol, luteolin (If) (Williams et al. 1981),

C-Glycosylflavone: isovitexin 7-glucoside (rz) (Phouphas 1956)

Caladium bicolor (Aiton) Vent.

Anthocyanin: cyanidin 3-rutinoside (If),

Flavonol: quercetin (If) (Williams et al. 1981)

Calla palustris L.

Flavan and Proanthocyanidin: procyanidin (If)

(Williams et al. 1981)

Carlephyton glaucophyllum Bogner

Flavan and Proanthocyanidin: procyanidin (If)

(Williams et al. 1981)

Carlephyton madagascariense Jum. Flavan and Proanthocyanidin: procyanidin (If) (Williams et al. 1981)

Cercestis afzelii Schott

Flavan and Proanthocyanidin: procyanidin (If) (Williams et al. 1981)

Cercestis congoensis Engl.

Flavan and Proanthocyanidin: procyanidin (If) (Williams et al. 1981)

Cercestis mirabilis (N.E.Br.) Bogner (as Rhektophyllum mirabile N.E.Br.) (Williams et al. 1981)

Anthocyanin: cyanidin 3-gentiobioside (pt), Flavan and Proanthocyanidin: propelargonidin (If) (Williams et al. 1981) 


\section{Colocasia esculenta (L.) Schott}

Anthocyanin: cyanidin 3-glucoside, cyanidin 3rhamnoside, pelargonidin 3-glucoside (cr) (Chan Jr. \& Kao-Jao 1977),

Flavone: chrysoeriol 7-hexoside, chrysoeriol 7rhamnosyl-(1 $\rightarrow 6)$-hexoside, luteolin 7-glucoside, luteolin 7-rutinoside, luteolin 7-sophoroside (If, st) (Iwashina et al. 1999, Leong et al. 2010, Ferreres et al. 2012, Li et al. 2014),

Flavan and Proanthocyanidin: procyanidin (If), Flavonol: quercetin (If) (Williams et al. 1981), C-Glycosylflavone: apigenin 6-C-hexoside-6"'hexoside, apigenin 8- $C$-pentoside- 2 "'-hexoside, apigenin 6- $C$-hexoside- 2 "'-hexoside- $8-C$ pentoside, apigenin 6- $C$-pentoside- 8 - $C$-hexoside7-hexoside, apigenin 6- $C$-pentoside-8- $C$ hexoside-2"'-hexoside, chrysoeriol 6- $C$-hexoside, chrysoeriol 8-C-hexoside, chrysoeriol 6-Chexoside-8- $C$-pentoside, diosmetin 6 - $C$-hexoside$8-C$-pentoside, isoschaftoside, isovitexin, isovitexin $4^{\prime}$-glucoside, isoorientin, luteolin $6-C$ hexoside- 6 "'-hexoside, luteolin 6 - $C$-hexoside- 3 " hexoside-8-C-pentoside, luteolin 6,8-di- $C$ hexoside, luteolin 6-C-hexoside-2"'-pentoside, luteolin 6-C-hexoside-8-C-pentoside, luteolin 6- $C$ pentoside-8- $C$-hexoside, orientin, orientin 7 glucoside, schaftoside, vicenin-2, vitexin, vitexin X"'-glucoside (If, st) (Iwashina et al. 1999, Leong et al. 2010, Ferreres et al. 2012, Li et al. 2014)

Cryptocoryne albida R.Parker C-Glycosylflavone: vitexin 2"'-glucoside-6"'-Esinapate, vitexin 2"-glucoside (If) (Franke et al. 2006)

Cryptocoryne crispatula Engl.

C-Glycosylflavone: vitexin 2"'-glucoside (If) (Franke et al. 2006)

Cryptocoryne pontederiifolia Schott C-Glycosylflavone: vitexin 2"'-glucoside-6"'-Esinapate, vitexin 2"'-glucoside (If) (Franke et al. 2006)

Cryptocoryne retrospiralis (Roxb.) Kunth C-Glycosylflavone: vitexin 2"'-glucoside (If) (Franke et al. 2006)

Cryptocoryne spiralis (Retz.) Fisch. ex Wydler C-Glycosylflavone: vitexin 2"-glucoside (If) (Franke et al. 2006)

Cryptocoryne usteriana Engl. C-Glycosylflavone: vitexin 2"'-glucoside-6"'-Esinapate, vitexin 2"'-glucoside (If) (Franke et al. 2006)
Cryptocoryne vietnamensis I.hertel \& H.Mühlberg

C-Glycosylflavone: vitexin 2"'-glucoside (If)

(Franke et al. 2006)

Cryptocoryne wendtii de Wit

Flavone: chrysoeriol, luteolin (If) (Williams et al. 1981)

Cryptocoryne xwillisii Reitz

C-Glycosylflavone: vitexin 2"'-glucoside (If)

(Franke et al. 2006)

Culcasia scandens P.Beauv. (as Culcasia saxatilis

A.Chev.) (Williams et al. 1981)

C-Glycosylflavone: vitexin 7-sulfate (If) (Williams et al. 1981)

Dracunculus canariensis Kunth

Anthocyanin: cyanidin 3-glucoside, cyanidin 3rutinoside (sp),

Flavone: chrysoeriol, luteolin (If) (Williams et al. 1981)

Dracunculus vulgaris Schott (as Arum dracunculus

L.) (Proliac et al. 1992)

C-Glycosylflavone: isoorientin, orientin, vitexin

(If) (Proliac et al. 1992)

Eminium regelii Vved.

Flavone: luteolin (If),

Flavonol: quercetin (If) (Silybayeva et al. 2014)

Eminium spiculatum (Blume) Schott

Flavone: chrysoeriol 7-glucoside, luteolin, luteolin 7-glucoside (If),

C-Glycosylflavone: isoorientin, vitexin (If) (Afifi \& Abu-Dahab 2012)

Helicodiceros muscivorus (L.f.) Engl.

Anthocyanin: cyanidin 3-rutinoside (sp) (Williams et al. 1981)

Homalomena pendulata (Blume) Bakh.f. (as Homalomena coerulescens Jungh. ex Miq.)

(Williams et al. 1981)

Flavan and Proanthocyanidin: procyanidin (If)

(Williams et al. 1981)

Homalomena rubescens (Roxb.) Kunth

Anthocyanin: cyanidin 3-glucoside (If, pt),

Flavonol: quercetin (If) (Williams et al. 1981)

Peltandra virginica (L.) Schott

Flavan and Proanthocyanidin: procyanidin (If),

Flavonol: quercetin (If) (Williams et al. 1981) 
Philodendron auriculatum Standl. \& L.O.Williams Flavan and Proanthocyanidin: procyanidin (If) (Williams et al. 1981)

Philodendron crassinervium Lindl.

Flavan and Proanthocyanidin: procyanidin (If), Flavonol: isorhamnetin, kaempferol, quercetin (If) (Williams et al. 1981)

Philodendron erubescens K.Koch \& Augustin Anthocyanin: cyanidin 3-glucoside, cyanidin 3rutinoside (pt, sp, sx),

Flavonol: quercetin (If) (Williams et al. 1981)

Philodendron fendleri K. Krause

Anthocyanin: cyanidin, delphinidin (sp) (Forsyth \& Simmonds 1954)

Philodendron giganteum Schott

Anthocyanin: cyanidin (sp) (Forsyth \& Simmonds 1954)

Philodendron goeldii G.M.Barroso

Flavan and Proanthocyanidin: procyanidin (If) (Williams et al. 1981)

Philodendron hastatum K.Koch \& Sello

Anthocyanin: cyanidin 3-glucoside, cyanidin 3rutinoside (sp),

Flavonol: quercetin 3-glucoside (sp) (Alfa et al. 1987)

Philodendron hederaceum (Jacq.) Schott (as Philodendron scandens K.Koch \& F.Sello subsp. prieurianum (Schott) G.S.Bunting) (Williams et al. 1981)

Flavan and Proanthocyanidin: procyanidin (If) (Williams et al. 1981)

Philodendron hederaceum as Philodendron scandens subsp. scandens in Williams et al. (1981) Flavan and Proanthocyanidin: procyanidin (If) (Williams et al. 1981)

Philodendron imbe Schott \& Kunth Flavan and Proanthocyanidin: procyanidin (If) (Williams et al. 1981)

Philodendron insigne Schott

Anthocyanin: cyanidin 3-rutinoside (If), Flavan and Proanthocyanidin: procyanidin (If), Flavonol: kaempferol, quercetin (If) (Williams et al. 1981)

Philodendron latifolium K.Koch

Anthocyanin: cyanidin, delphinidin (sp) (Forsyth \& Simmonds 1954)
Philodendron leal-costae Mayo \& G.M.Barroso

Flavan and Proanthocyanidin: procyanidin (If),

Flavonol: kaempferol, quercetin (If) (Williams et al. 1981)

Philodendron linnaei Kunth

Anthocyanin: cyanidin 3-rutinoside (If), Flavan and Proanthocyanidin: procyanidin (If), Flavonol: kaempferol, quercetin (If) (Williams et al. 1981)

Philodendron longilaminatum Schott

Flavan and Proanthocyanidin: procyanidin (If), Flavonol: kaempferol, quercetin (If) (Williams et al. 1981)

Philodendron melanochrysum Linden \& André Flavan and Proanthocyanidin: procyanidin (If) (Williams et al. 1981)

Philodendron melinonii Brongn. \& Regel

Anthocyanin: cyanidin 3-glucoside (If), Flavonol: isorhamnetin, quercetin (If) (Williams et al. 1981)

Philodendron ornatum Schott

Anthocyanin: cyanidin 3-glucoside (If), C-Glycosylflavone: isovitexin 7-sulfate, vitexin 7sulfate (If) (Williams et al. 1981)

Philodendron pachyphyllum K.Krause

Flavonol: quercetin (If) (Williams et al. 1981)

Philodendron pedatum (Hook.) Kunth

Flavan and Proanthocyanidin: procyanidin (If), Flavonol: quercetin (If) (Williams et al. 1981)

Philodendron radiatum Schott

Flavan and Proanthocyanidin: procyanidin (If)

(Williams et al. 1981)

Philodendron saxicola K.Krause

Flavan and Proanthocyanidin: procyanidin (If), Flavonol: isorhamnetin 3-glucoside, isorhamnetin 3-rutinoside, quercetin 3-glucoside, quercetin 3-rutinoside (If),

C-Glycosylflavone: apigenin di-C-glycoside, luteolin di-C-glycoside, luteolin $C$-glycoside (If) (Williams et al. 1981)

Philodendron simsii (Hook.) Sweet ex Kunth

Flavan and Proanthocyanidin: procyanidin (If) (Williams et al. 1981)

Philodendron smithii Engl.

Flavan and Proanthocyanidin: procyanidin (If), 
C-Glycosylflavone: isoschaftoside, schaftoside (If) (Williams et al. 1981)

Philodendron squamiferum Poepp.

Anthocyanin: cyanidin 3-rutinoside (pt) (Williams et al. 1981)

Philodendron undulatum Engl. (as Philodendron eichleri Engl.) (Williams et al. (1981)

Flavan and Proanthocyanidin: procyanidin (If), C-Glycosylflavone: isoorientin, isoschaftoside, orientin, schaftoside (If) (Williams et al. 1981)

Philodendron verrucosum L. Mathieu ex Schott Anthocyanin: cyanidin 3-glucoside, cyanidin 3rutinoside (If, pt) (Williams et al. 1981)

Pinellia tripartita (Blume) Schott

Anthocyanin: cyanidin 3-rutinoside (st) (Williams et al. 1981)

Pistia stratiotes L.

Anthocyanin: cyanidin 3-glucoside (wp) (Zennie \& McClure 1977, Tripathi et al. 2016), Flavone: chrysoeriol 4'-glucoside, luteolin, luteolin 7-glycoside (wp) (Zennie \& McClure 1977, Liu et al. 2008, Tripathi et al. 2016), C-Glycosylflavone: apigenin 6,8-di-C-glycoside, luteolin 6,8-di-C-glycoside, orientin, vitexin (wp) (Zennie \& McClure 1977, Tripathi et al. 2016)

Sauromatum giganteum (Engl.) Cusimano \& Hett. (as Typhonium giraldii (Baroni) Engl.) (Williams et al. 1981)

Anthocyanin: cyanidin 3-rutinoside (st), Flavone: chrysoeriol, luteolin (If) (Williams et al. 1981)

Stylochaeton borumensis N.E.Br.

Flavonol: quercetin (If) (Williams et al. 1981)

Stylochaeton lancifolius Kotschy \& Peyr.

Anthocyanin: cyanidin 3-rutinoside (st), Flavan and Proanthocyanidin: procyanidin (If), Flavonol: kaempferol, quercetin (If) (Williams et al. 1981)

Synandrospadix vermitoxicus (Griseb.) Engl. C-Glycosylflavone: isoorientin, isovitexin, isovitexin 7-glucoside, orientin, vitexin (If) (Sosa et al. 1978)

Syngonium auritum (L.) Schott

Flavan and Proanthocyanidin: procyanidin (If) (Williams et al. 1981)

\section{Syngonium podophyllum Schott \\ C-Glycosylflavone: isoschaftoside (If) (Gomes et al. 2014)}

Typhonium flagelliforme (Lodd.) Blume C-Glycosylflavone: isovitexin (If) (Farida et al. 2012)

Typhonodorum lindleyanum Schott

Anthocyanin: cyanidin 3-glucoside, cyanidin 3rutinoside (If),

Flavan and Proanthocyanidin: procyanidin (If)

(Williams et al. 1981)

Xanthosoma brasiliense (Desf.) Engl.

Flavonol: quercetin (If) (Williams et al. 1981)

Xanthosoma helleborifolium (Jacq.) Schott

Anthocyanin: cyanidin 3-rutinoside, pelargonidin 3-rutinoside (st) (Williams et al. 1981)

Xanthosoma sagittifolium (L.) Schott Flavan and Proanthocyanidin: procyanidin (If) (Williams et al. 1981)

Xanthosoma sagittifolium (as Xanthosoma violaceum Schott) (Williams et al. 1981, Picerno et al. 2003)

Anthocyanin: cyanidin 3-rutinoside (If) (Williams et al. 1981),

C-Glycosylflavone: apigenin 6-C-glucoside-8-Capiofuranoside, isovitexin, isovitexin $6^{\prime \prime}$ glucoside, isovitexin $4^{\prime}$-rhamnoside, vitexin, vicenin-2 (If) (Picerno et al. 2003)

Zantedeschia aethiopica (L.) Spreng.

Flavan and Proanthocyanidin: procyanidin (fl),

Flavone: apigenin, luteolin ( $\mathrm{fl}$ ),

Flavonol: kaempferol, quercetin (fl) (Martens et al., 2003),

C-Glycosylflavone: isoorientin, isovitexin, orientin, swertiajaponin, swertisin, vitexin (fl, If, sp) (Martens et al. 2003, Luzzatto et al. 2007, Nakayama et al. 2015)

\section{FLAVONOIDS IN THE FAMILY ACORACEAE}

Although the genus Acorus is now erected to its own family, it used to be a member of the Araceae. Of two Acorus species, A. calamus was surveyed for flavonoids and a $C$-glycosylflavone, lucenin-2 (Figure 50), was found (Él'yashevich et al. 1974). 


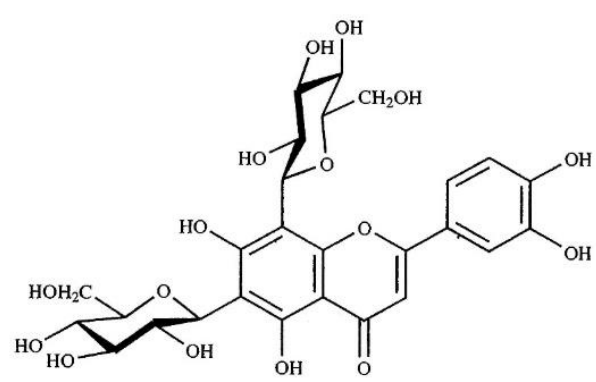

Figure 50. Lucenin-2

Table 8. Reports on the flavonoids from the species of the family Acoraceae

\section{Acorus calamus L. \\ C-Glycosylflavone: Lucenin-2 (ap) (Él'yashevich et al. 1974)}

The occurrence of flavonoid classes among the genera of Araceae and the related Acoraceae was shown in Table 9. The presence of anthocyanins, flavones, C-glycosylflavones, flavonols, flavan and proanthocyanidins, flavanones and $\quad C$-glycosylflavanone were recognized. However, dihydroflavonol, chalcone, dihydrochalcone, aurone, isoflavonoid, and so on were not reported as far as I know. C-Glycosylflavones seem to be major flavonoids in the Araceae. Although Williams et al. (1981) showed the presence of $C$ - glycosylflavones in almost species, they were not isolated and characterized. Of six subfamilies of the Araceae, C-glycosylflavones were not found in Gymnostachydoideae and Orontioideae. Instead, flavonols were major flavonoids in both subfamilies. In subfamily Lemnoideae, flavones and flavonols occurred in the genera Lemna, Spirodela and Wolffia. On the other hand, flavonols was absent in Wolffillea. Flavan and proanthocyanidins were also major flavonoids and present in 46 species. They were procyanidins except for propelargonidin in Cercestis mirabilis (as Rhektophyllum mirabile), but insufficiently identified (Williams et al. 1981). Subfamily Lemnoideae was not surveyed for flavan and proanthocyanidins except for Spirodela punctata. Flavanones were isolated from Spirodela polyrrhyza in subfamily Lemnoideae and Anthurium binotii in subfamily Pothodeae, and identified as eriodictyol and hesperetin 7-glucosides (Quiao et al. 2011), and hensperetin 7-rutinoside (Brunswik 1921), respectively. C-Glycosylflavanone was isolated from Spirodela polyrrhyza and identified as naringenin 6,8-di-C-glucoside (Quiao et al. 2011). The family Araceae s.l. (including Lemnaceae) consists of more than 3645 species of ca. 144 genera (Boyce \& Croat 2011 onwards). However, flavonoids were surveyed in 146 species of 48 genera only. Further flavonoid survey must be performed in the Araceae. 
Table 9. The occurrence of flavonoid classes among the genera of Araceae

\begin{tabular}{|c|c|c|c|c|c|c|c|c|}
\hline & No. & An & $\mathrm{Fv}$ & $\mathrm{Fn}$ & CG & $\mathrm{FP}$ & $\mathrm{Fa}$ & $\mathrm{Cf}$ \\
\hline \multicolumn{9}{|c|}{ Subfamily Gymnostachydoideae } \\
\hline Gymnostachys & 1 & & & 1 & & & & \\
\hline \multicolumn{9}{|c|}{ Subfamily Orontioideae } \\
\hline Lysichiton & 1 & & & 1 & & & & \\
\hline Orontium & 1 & & & 1 & & & & \\
\hline Symplocarpus & 2 & 1 & & 2 & & & & \\
\hline \multicolumn{9}{|c|}{ Subfamily Lemnoideae } \\
\hline Lemna & 8 & 3 & 4 & & 8 & & & \\
\hline Spirodela & 3 & 3 & 3 & 3 & 3 & 1 & 1 & 1 \\
\hline Wolffia & 5 & & 3 & 2 & 3 & & & \\
\hline Wolffillea & 3 & & & 3 & & & & \\
\hline \multicolumn{9}{|c|}{ Subfamily Pothoideae } \\
\hline Anthurium & 22 & 16 & 2 & 5 & 3 & 8 & 1 & \\
\hline Pothos & 1 & & 1 & & 1 & & & \\
\hline \multicolumn{9}{|c|}{ Subfamily Monsteroideae } \\
\hline Scindapsus & 1 & & 1 & 1 & & & & \\
\hline \multicolumn{9}{|l|}{ Subfamily Lasioideae } \\
\hline Dracontium & 1 & 1 & & & 1 & & & \\
\hline Lasia & 1 & & & 1 & 1 & & & \\
\hline \multicolumn{9}{|l|}{ Subfamily Aroideae } \\
\hline Aglaonema & 1 & & & & & 1 & & \\
\hline Alocasia & 7 & 6 & & 4 & & 2 & & \\
\hline Amorphophallus & 5 & 5 & 1 & 3 & 3 & & & \\
\hline Anchomanes & 2 & 1 & & & & 1 & & \\
\hline Anubias & 1 & & & & & 1 & & \\
\hline Apobalis & 1 & 1 & & 1 & & 1 & & \\
\hline Arisaema & 2 & 1 & & & 1 & & & \\
\hline Arisarum & 1 & & & & 1 & & & \\
\hline Arophyton & 1 & & & & & 1 & & \\
\hline Arum & 6 & 1 & 6 & 3 & 5 & & & \\
\hline Asterostigma & 1 & 1 & & & 1 & & & \\
\hline Biarum & 1 & & 1 & & 1 & & & \\
\hline Caladium & 1 & 1 & & 1 & & & & \\
\hline Calla & 1 & & & & & 1 & & \\
\hline Carlephyton & 2 & & & & & 2 & & \\
\hline Cercestis & 3 & 1 & & & & 3 & & \\
\hline Colocasia & 1 & 1 & 1 & 1 & 1 & 1 & & \\
\hline Cryptocoryne & 9 & & 1 & & 8 & & & \\
\hline Culcasia & 1 & & & & 1 & & & \\
\hline Dracunculus & 2 & 1 & 1 & & 1 & & & \\
\hline Eminium & 2 & & 2 & 1 & 1 & & & \\
\hline Helicodiceros & 1 & 1 & & & & & & \\
\hline Homalomena & 2 & 1 & & 1 & & 1 & & \\
\hline Peltandra & 1 & & & 1 & & 1 & & \\
\hline Philodendron & 26 & 11 & & 11 & 4 & 16 & & \\
\hline Pinellia & 1 & 1 & & & & & & \\
\hline Pistia & 1 & 1 & 1 & & 1 & & & \\
\hline Sauromatum & 1 & 1 & 1 & & & & & \\
\hline Stylochaeton & 2 & 1 & & 2 & & 1 & & \\
\hline Synandrospadix & 1 & & & & 1 & & & \\
\hline Syngonium & 2 & & & & 1 & 1 & & \\
\hline Typhonium & 1 & & & & 1 & & & \\
\hline Typhonodorum & 1 & 1 & & & & 1 & & \\
\hline Xanthosoma & 3 & 2 & & 1 & 1 & 1 & & \\
\hline Zanthodeschia & 1 & & 1 & 1 & 1 & 1 & & \\
\hline \multicolumn{9}{|l|}{ Family Acoraceae } \\
\hline Acorus & 1 & & & & 1 & & & \\
\hline Total & 146 & 64 & 30 & 51 & 55 & 46 & 2 & 1 \\
\hline
\end{tabular}

No. $=$ number of the species from which flavonoids were reported. $A n=$ anthocyanins, $F v=$ flavones, $F n=$ flavonols, $C G=C$ glycosylfavones, $\mathrm{FP}=$ flavan and proanthocyanidins, $\mathrm{Fa}=$ flavanones and $\mathrm{Cf}=\mathrm{C}$-glycosylflavanone. 


\section{ACKNOWLEDGMENTS}

Author thanks to Dr. Sri Rahayu (Bogor Botanic Gardens, Indonesia) for invitation of submitting to the Journal, and also Dr. Joko Ridho Witono (Bogor Botanic Gardens) for reviewing of the manuscript.

\section{REFERENCES}

Afifi FU, Khalil E, Abdalla S. 1999. Effect of isoorientin isolated from Arum palaestinum on uterine smooth muscle of rats and guinea pigs. Journal of Ethnopharmacology 65: 173177.

Afifi FU, Abu-Dahab R. 2012. Phytochemical screening and biological activities of Eminium spiculatum (Blume) Kuntze (family Araceae). Natural Product Research 26(9): 878-882.

Afifi FU, Kasabri V, Litescu SC, Abaza IM. 2016. In vitro and in vivo comparison of the biological activities of two traditionally and widely used Arum species from Jordan: Arum dioscoridis Sibth \& Sm. and Arum palaestinum Boiss. Natural Product Research 30(16): 1777-1786.

Akhtar TA, Lees HA, Lampi MA, Enstone D, Brain RA, Greenberg BM. 2010. Photosynthetic redox imbalance influences flavonoid biosynthesis in Lemna gibba. Plant, Cell and Environment 33: 1205-1219.

Alfa M, Maccarone E, d'Alcontres GS. 1987. The anthocyanins of Philodendron hastatum. Annali di Chimica 77: 691-695.

Andersen $\varnothing \mathrm{M}$, Markham KR (eds.). 2006. Flavonoids. Chemistry, Biochemistry and Applications. Taylor and Francis, Boca Raton.

Aquino R, Morelli S, Lauro MR, Abdo S, Saija A, Tomaino A. 2001. Phenolic constituents and antioxidant activity of an extract of Anthurium versicolor leaves. Journal of Natural Products 64(8): 1019-1023.

Bai HH, Wang NN, Mi J, Yang T, Fang DM, Wu LW, Zhao H, Li GY. 2018. Hydroxycinnamoylmalated flavone $C$-glycosides from Lemna japonica. Fitoterapia 124: 211-216.

Boyce PC, Croat TB. (2011 onwards). The Überlist of Araceae, totals for published and estimated number of species in Aroid Genera. http://www.aroid.org/genera/180211uberli st.pdf
Brunswik H. 1921. Über Hesperidinsphärite im lebenden Hauptgewebe von Anthurium binotii Linden. Berichte der Deutschen Botanischen Gesellschaft 39: 208-212.

Chang N, Collins RP, Knaak LE. 1970. Anthocyanins in Symplocarpus foetidus (L.) Nutt. (Araceae). Botanical Journal of the Linnean Society 63: 95-96.

Chan Jr. HT, Kao-Jao THC. 1977. Anthocyanin composition of taro. Journal of Food Science 42: 19-21.

Clark BR, Suzuki JY, Bliss BJ, Borris RP. 2012. Flavone C-glycosides from Anthurium andraeanum. Natural Product Communications 7(6): 747748.

Cody V, Middleton Jr. E, Harborne JB (eds.). 1986. Plant Flavonoids in Biology and Medicine. Biochemical, Pharmacological, and Structure-activity Relationship. Alan R. Liss, Inc. New York.

Cody V, Middleton Jr. E, Harborne JB, Beretz A (eds.). 1988. Plant Flavonoids in Biology and Medicine II. Biochemical, Cellular, and Medicinal Properties. Alan R. Liss, Inc. New York.

Du SS, Lei N, Xu YC, Wei LX. 2005. Study on flavonoids of Arisaema erubescens. Journal of Chinese Pharmaceutical Journal 40(19): 1457-1459.

Du SS, Zhang HM, Bai CQ, Wang CF, Liu QZ, Liu ZL, Wang YY, Deng ZW. 2011. Nematocidal flavone-C-glycosides against the root-knot nematode (Meloidogyne incognita) from Arisaema erubescens tubers. Molecules 16: 5079-5086.

Él'yashevich EG, Drozd GA, Koreshchuk KE, Bezuglaya NI, Manych VI, Shestak LI. 1974. Acorus calamus - A new source of $C$ diglycosides. Chemistry of Natural Compounds 10: 96-97.

Farid MM, Hussein SR, Ibrahim LF, El Desouky MA, Elsayed AM, El Oqlah AA, Saker MM. 2015. Cytotoxic activity and phytochemical analysis of Arum palaestinum Boiss. Asian Pacific Journal of Tropical Biomedicine 5(11): 944947.

Farida Y, Wahyudi PS, Wahono S, Hanafi M. 2012. Flavonoid glycoside from the ethyl acetate extract of Keladi tikus Typhonium flagelliforme (Lodd) Blume leaves. Asian 
Journal of Natural and Applied Sciences 1(4): 16-21.

Ferreres F, Gonçalves RF, Gil-Izquierdo A, Valentão P, Silva AMS, Silva JB, Santos D, Andrade PB. 2012. Further knowledge on the phenolic profile of Colocasia esculenta (L.) Schott. Journal of Agricultural and Food Chemistry 60: 7005-7015.

Forsyth WGC, Simmonds NW. 1954. A survey of the anthocyanins of some tropical plants. Proceedings of the Royal Society of London, Series B 142: 549-564.

Franke K, Hoffmann M, Schmidt J, Porzel A, Arnold N, Wessjohann L. 2006. 2"-O-Glucosylvitexin, a chemotaxonomic marker for the genus Cryptocoryne (Araceae). Biochemical Systematics and Ecology 34: 546-548.

Gallori S, Giccherini C, Bilia AR, Mulinacci N, Vincieri FF. 2004. Identification of anthocyanins in Amorphophallus titanum (Becc.) Becc. Chromatographia 59(9/10): 647-651.

Gomes ACC, da Silva Sampaio L, da Silva PA, Lamas ME, Sakuragui CM, Junior CBB, Simas NK, Kuster RM. 2014. In vitro effect of isoschaftoside isolated from Syngonium podophyllum on pig kidney $\mathrm{Na}^{+}, \mathrm{K}^{+}$-atpase. Quimica Nova 37(10): 1606-1609.

Harborne JB, Mabry TJ, Mabry H (eds.). 1975. The Flavonoids. Chapman and Hall, London.

Harborne JB, Mabry TJ (eds.). 1982. The Flavonoids: Advances in Research. Chapman and Hall, London.

Harborne JB (ed.). 1988. The Flavonoids. Advances in Research since 1980. Chapman and Hall, London.

Harborne JB. (ed.). 1994. The Flavonoids. Advances in Research since 1986. Chapman and Hall, London.

Hong Van NT, Minh CV, De Leo M, Siciliano T, Braca A. 2006. Secondary metabolites from Lasia spinosa (L.) Thw. (Araceae). Biochemical Systematics and Ecology 34: 882-884.

Iwashina T, Konishi T, Takayama A, Fukuda M, Ootani S. 1999. Isolation and identification of the flavonoids in the leaves of taro. Annals of the Tsukuba Botanical Garden 18: 71-74.

Iwashina T. 2000. The structure and distribution of the flavonoids in plants. Journal of Plant Research 113: 287-299.

Iwashina T. 2003. Flavonoid function and activity to plants and other organisms. Biological Sciences in Space 17(1): 24-44.

Iwashina T, Peng $\mathrm{Cl}$, Kokubugata G. 2010. Flavone Oand $C$-glycosides from Pothos chinensis (Araceae). Bulletin of the National Museum of Nature and Science, Series B 36(1): 27-32.

Iwashina T, Uehara A, Kitajima J, Yukawa T. 2015. Anthocyanins and other flavonoids from Amorphophallus titanum having largest inflorescence in plant kingdom, and other two species. Bulletin of the National Museum of Nature and Science, Series B 41(1): 33-44.

Iwashina T, Destri, Rahayu S, Tsutsumi C, Yuzammi, Mizuno T, Widyatmoko D. 2020. Flavonoids and xanthones from the leaves of Amorphophallus titanum (Araceae). Biochemical Systematics and Ecology. DOI: https://doi.org/10.1016/j.bse.2020.104036

I wata RY, Tang CS, Kamemoto H. 1979. Anthocyanins of Anthurium andreanum Lindl. Journal of the American Society for Horticultural Science 104(4): 464-466.

Jurd L, Geissman TA, Seikel MK. 1957. The flavonoid constituents of Spirodela oligorrhiza. II. The flavone constituents. Archives of Biochemistry and Biophysics 67: 284-297.

Khan A, Rahman M, Islam MS. 2008. Antibacterial, antifungal and cytotoxic activities of 3,5diacetyltambulin isolated from Amorphophallus campanulatus Blume ex Decne. Daru 16(4): 239-244.

Kim J, Lee I, Seo J, Jung MY, Kim Y, Yim N, Bae K. 2010. Vitexin, orientin and other flavonoids from Spirodela polyrrhiza inhibit adipogenesis in 3T3-L1 cells. Phytotherapy Research 24: 1543-1548.

Koleva M. 1982. Assessment of the polyphenol content in Arum orientale M.B. leaves. Problemi na Farmatsiiata 10: 53-57.

Koleva M. 1984. Assessment of the polyphenol composition of Arum orientale M.B. Problemi na Farmatsiiata 12: 35-38.

Krause J, Strack D. 1979. Malonyl cyanidin 3monoglucoside in Spirodela polyrrhiza (L.) Schleiden. Zeitschrift für Pflanzenphysiologie 95: 183-187.

Lei X, Feng Y, Liang S, Zheng XW. 2014. Chemical components of the tuber of Alocasia cucullata. Chemistry of Natural Compounds 
50(1): 133-134.

Leong $\mathrm{ACN}$, Kinjo $\mathrm{Y}$, Tako $\mathrm{M}$, Iwasaki $\mathrm{H}$, Oku $\mathrm{H}$, Tamaki H. 2010. Flavonoid glycosides in the shoot system of Okinawa Taumu (Colocasia esculenta S.). Food Chemistry 119: 630-635.

Li C, Yang G, Huang S, Lü D, Wang C, Chen J, Yin J. 2013. Characterization of flavonoids in Anthurium spathes and their contribution to spathe colouration. Journal of Horticultural and Biotechnology 88(2): 208-215.

Li HM, Hwang SH, Kang BG, Hong JS, Lim SS. 2014. Inhibitory effects of Colocasia esculenta (L.) Schott constituents on aldose reductase. Molecules 19: 13212-13224.

List PH,. Freud B. 1968. Geruchssoffe der Stinkmorchel, Phallus impudicus L. 18. Mitteilung über Pilzinhaltsstoffe. Planta Medica (Supplement) 123-132.

Liu HW, He LY, Gao JM, Ma YB, Zhang XM, Peng H, Chen JJ. 2008. Chemical constituents from the aquatic weed Pistia stratiotes. Chemistry of Natural Compounds 44(2): 236-238.

Liu X, Wang CY, Shao CL, Wei YX, Wang BG, Sun LL, Zheng CJ, Guan HS. 2009. Chemical constituents from Sargassum pallidum (Turn) C.Agardh. Biochemical Systematics and Ecology 37: 127-129.

Luzzatto T, Golan A, Yishay M, Bilkis I, Ben-Ari J, Yedidia I. 2007. Priming of antimicrobial phenolics during induced resistance response towards Pectobacterium carotovorum in the ornamental monocot calla lily. Journal of Agricultural and Food Chemistry 55(25): 10315-10322.

Markham KR, Williams CA. 1980. CArabinosylapigenin methyl ethers from Asterostigma riedelianum. Phytochemistry 19: 2789-2791.

Martens S, Knott J, Seitz CA, Janvari L, Yu SN, Forkmann G. 2003. Impact of biochemical pre-studies on specific metabolic engineering strategies of flavonoid biosynthesis in plant tissues. Biochemical Engineering Journal 14: 227-235.

McClure JW, Alston RE. 1966. A chemotaxonomic study of Lemnaceae. American Journal of Botany 53(9): 849-860.

McClure JW. 1968. Photocontrol of Spirodela intermedia flavonoids. Plant Physiology 43: 193-200.
Nakayama M, Koshioka M, Kondo T, Imizu K. 2015. Flavone $C$-glucosides responsible for yellow pigmentation induced by low temperature in bracts of Zanthodeschia aethiopics. Natural Product Communications 10(3): 425-427.

Ohba R, Igarashi K, Tsukui A. 2000. Anthocyanins. Food Color with Health Benefits. Kenpousha, Tokyo.

Pagani F. 1982. Fitoconstituenti dell'Arisarum vulgare O. Targ.-Tozz. (Araceae). Bollettino Chimico Farmaceutico 121: 460-464.

Phouphas MC. 1956. Sur la presence de saponaroside chez certaines Aracées. Comptes Rendus de l'Académie des Sciences, Paris 242: 1641-1643.

Picerno P, Mencherini T, Lauro MR, Barbato F, Aquino R. 2003. Phenolic constituents and antioxidant properties of Xanthosoma violaceum leaves. Journal of Agricultural and Food Chemistry 51(22): 6423-6428.

Proliac A, Chaboud A, Raynaud J. 1992. Isolement et identification de trois C-glycosylflavones dans les tiges feuillées d'Arum dracunculus. Die Pharmazie 47(8): 646-647.

Quiao X, He W, Xiang C, Han J, Wu L, Guo D, Ye M. 2011. Qualitative and quantitative analysis of flavonoids in Spirodela polyrrhiza by highperformance liquid chromatography coupled with mass spectrometry. Phytochemical Analysis 22: 475-483.

Reznik H, Menschick R. 1969. Flavonoide in Sommergliedern und Winterknospen von Spirodela polyrrhiza (L.) Schleid. Zeitschrift für Pflanzenphysiologie 61: 348-349.

Rice-Evans CA, Packer L. 1998. Flavonoids in Health and Disease. Marcel Dekker, Inc., New York.

Saunders JA, McClure JW. 1976. The distribution of flavonoids in chloroplasts of twenty five species of vascular plants. Phytochemistry 15(5): 809-810.

Sharstry RA, Biradar SM, Mahadevan KM, Habbu PV. 2010. Isolation and characterization of secondary metabolite from Amorphophallus paeoniifolius for hepatoprotective activity. Research Journal of Pharmaceutical, Biological and Chemical Sciences 1(4): 429437.

Silybayeva B, Zharykbasova K, Musabayeva B, Omargalieva N, Kaygusuz O. 2014. Biologically active agents of Eminium regelii 
leaves and tubers grown in Kazakhstan. Bothalia Journal 44(5): 156-160.

Sosa VE, Oberti JC, Juliani HR. 1978. Flavonoids of Synandrospadix vermitoxicus. Lloydia 41(3): 277-278.

Tripathi P, Kumar R, Sharma AK, Mishra A, Gupta R. 2016. Pistia stratiotes (Jalkumbhi). Pharmacognosy Reviews 4(8): 153-160.

Ueno N, Takemura E, Hayashi K. 1969. Additional data for the paperchromatographic survey of anthocyanins in the flora of Japan (IV). Studies on anthocyanins, LXI. Botanical Magazine, Tokyo 82: 155-161.

Veen J. 1975. Preliminary studies of the flavonoid pattern of Lemna gibba L. and Lemna minor L. Aquatic Botany 1: 417-421.

Vladimirova IN, Georgiyants VA. 2013. Biologically active compounds from Lemna minor S.F. Gray. Pharmaceutical Chemistry Journal 47(11): 599-601.

Wallace JW, Alston RE. 1966. C-Glycosylation of flavonoids. Plant and Cell Physiology 7: 699700.

Wallace JW, Mabry TJ, Alston RE. 1969. On the biogenesis of flavone $O$-glycosides and $C$ glycosides in the Lemnaceae. Phytochemistry 8(1): 93-99.

Wallace JW. 1975. Biosynthetic studies on flavones and C-glycosylflavones: B-ring oxidation patterns. Phytochemistry 14(8): 1765-1768.

Wang N, Xu G, Fang Y, Yang T, Zhao H, Li G. 2014a. New flavanol and cycloartane glucosides from Landoltia punctata. Molecules 19: 6623-6634.

Wang N, Fang Y, Yang T, Li G, Zhao H. 2014b. Chemical constituents of Wolffia globosa. Chinese Journal of Applied and Environmental Biology 20(6): 1016-1019.

Whang WK, Lee MT. 1999. New flavonol glycosides from leaves of Symplocarpus renifolius. Archives of Pharmacal Research 22(4): 423429.

Williams CA, Harborne JB, Clifford HT. 1971. Flavonoid patterns in the monocotyledons. Flavonols and flavones in some families associated with the Poaceae. Phytochemistry 10(5): 1059-1063.

Williams CA, Harborne JB, Mayo SJ. 1981. Anthocyanin pigments and leaf flavonoids in the family Araceae. Phytochemistry 20(2): 217-234.

Zeng LM, Wang CJ, Su JY, Li D, Owen NL, Lu Y, Lu N, Zheng QT. 2001. Flavonoids from the red algae Acanthophora spicifera. Chinese Journal of Chemistry 19: 1097-1100.

Zennie TM, McClure JW. 1977. The flavonoid chemistry of Pistia stratiotes L. and the origin of the Lemnaceae. Aquatic Botany 3: 49-54. 